\title{
Entropy of Semiclassical Measures for Nonpositively Curved Surfaces
}

\author{
Gabriel Rivière
}

\begin{abstract}
We study the asymptotic properties of eigenfunctions of the Laplacian in the case of a compact Riemannian surface of nonpositive sectional curvature. To do this, we look at sequences of distributions associated to them and we study the entropic properties of their accumulation points, the so-called semiclassical measures. Precisely, we show that the Kolmogorov-Sinai entropy of a semiclassical measure $\mu$ for the geodesic flow $g^{t}$ is bounded from below by half of the Ruelle upper bound, i.e.

$$
h_{K S}(\mu, g) \geq \frac{1}{2} \int_{S^{*} M} \chi^{+}(\rho) \mathrm{d} \mu(\rho),
$$

where $\chi^{+}(\rho)$ is the upper Lyapunov exponent at point $\rho$. The main strategy is the same as in Rivière (Duke Math J, arXiv:0809.0230, 2008) except that we have to deal with weakly chaotic behavior.
\end{abstract}

\section{Introduction}

Let $M$ be a compact, connected, $\mathcal{C}^{\infty}$ riemannian manifold without boundary. For all $x \in M, T_{x}^{*} M$ is endowed with a norm $\|\cdot\|_{x}$ given by the metric over $M$. The geodesic flow $g^{t}$ over $T^{*} M$ is defined as the Hamiltonian flow corresponding to $H(x, \xi):=\frac{\|\xi\|_{x}^{2}}{2}$. This quantity corresponds to the classical kinetic energy in the case of the absence of potential. As any observable, this quantity can be quantized via pseudodifferential calculus and the quantum operator corresponding to $H$ is $-\frac{\hbar^{2} \Delta}{2}$ where $\hbar$ is proportional to the Planck constant and $\Delta$ is the Laplace Beltrami operator acting on $L^{2}(M)$. Our main concern in this article will be to study the asymptotic behavior, as $\hbar$ tends to 0 , of the following sequence of distributions:

$$
\forall a \in \mathcal{C}_{o}^{\infty}\left(T^{*} M\right), \quad \mu_{\hbar}(a)=\int_{T^{*} M} a(x, \xi) \mathrm{d} \mu_{\hbar}(x, \xi):=\left\langle\psi_{\hbar}, \mathrm{Op}_{\hbar}(a) \psi_{\hbar}\right\rangle_{L^{2}(M)},
$$


where $\mathrm{Op}_{\hbar}(a)$ is a $\hbar$-pseudodifferential operator of symbol $a$ [8] and $\psi_{\hbar}$ satisfies

$$
-\hbar^{2} \Delta \psi_{\hbar}=\psi_{\hbar}
$$

An accumulation point (as $\hbar \rightarrow 0$ ) of such a sequence of distribution $\mu_{\hbar}$ is called a semiclassical measure. Moreover, one knows that a semiclassical measure is a probability measure on $S^{*} M:=\left\{\|\xi\|_{x}^{2}=1\right\}$ which is invariant under the geodesic flow $g^{t}$ on $S^{*} M$. For manifolds of negative curvature, the geodesic flow on $S^{*} M$ satisfies strong chaotic properties (Anosov property, ergodicity of the Liouville measure) and as a consequence, it can be shown that almost all the sequences $\left(\mu_{\hbar}\right)_{\hbar \rightarrow 0}$ converge to the Liouville measure on $S^{*} M[7,20,23]$. This phenomenon is known as the quantum ergodicity property. A main challenge concerning this result would be to answer the Quantum Unique Ergodicity Conjecture [17], i.e. determine whether the Liouville measure is the only semiclassical measure or not (at least for manifolds of negative curvature).

In [2], Anantharaman used the Kolmogorov-Sinai entropy to derive properties of semiclassical measures on manifolds of negative curvature. ${ }^{1}$ In particular, she showed that the Kolmogorov-Sinai entropy of any semiclassical measure is positive. This result implies that the support of a semiclassical measure cannot be restricted to a closed geodesic, i.e. eigenfunctions of the Laplacian cannot concentrate only on closed geodesics in the high energy limit. In subsequent works, with Nonnenmacher and Koch, more quantitative lower bounds on the entropy of semiclassical measures were given [3,4].

\subsection{Kolmogorov-Sinai Entropy}

Let us recall a few facts about the Kolmogorov-Sinai (also called metric) entropy (see [22] or Appendix B for more details and definitions). It is a nonnegative number associated to a flow $\left(g^{t}\right)_{t}$ and to a $\left(g^{t}\right)_{t}$-invariant measure $\mu$, that estimates the complexity of $\mu$ with respect to this flow. For example, a measure carried by a closed geodesic will have entropy zero while the Liouville measure has large entropy. Recall also that a standard theorem of dynamical systems due to Ruelle [18] asserts that, for any invariant measure $\mu$ under the geodesic flow:

$$
h_{K S}(\mu, g) \leq \int_{S^{*} M} \sum_{j} \chi_{j}^{+}(\rho) \mathrm{d} \mu(\rho)
$$

with equality if and only if $\mu$ is the Liouville measure in the case of an Anosov flow [15]. In the previous inequality, the $\chi_{j}^{+}$denoted the positive Lyapunov exponents of $\left(S^{*} M, g^{t}, \mu\right)[6]$.

Regarding these properties, the main result of Anantharaman, Koch and Nonnenmacher was to show that, for a semiclassical measure $\mu$ on an Anosov manifold, one has

$$
h_{K S}(\mu, g) \geq \int_{S^{*} M} \sum_{j=1}^{d-1} \chi_{j}^{+}(\rho) \mathrm{d} \mu(\rho)-\frac{(d-1) \lambda_{\max }}{2} .
$$

\footnotetext{
${ }^{1}$ In fact, her result was about manifolds with Anosov geodesic flow, for instance manifolds of negative curvature.
} 
where $\lambda_{\max }:=\lim _{t \rightarrow \pm \infty} \frac{1}{t} \log \sup _{\rho \in S^{*} M}\left|d_{\rho} g^{t}\right|$ is the maximal expansion rate of the geodesic flow and the $\chi_{j}^{+}$'s are the positive Lyapunov exponents [6]. Compared with the original result from [2], this inequality gives an explicit lower bound on the entropy of a semiclassical measure. For instance, for manifolds of constant negative curvature, this lower bound can be rewritten as $\frac{d-1}{2}$. However, it can turn out that $\lambda_{\max }$ is a very large quantity and in this case, the previous lower bound can be negative (which would imply that it is an empty result). Combining these two observations [4], they were lead to formulate the conjecture that, for any semiclassical measure $\mu$, one has

$$
h_{K S}(\mu, g) \geq \frac{1}{2} \int_{S^{*} M} \sum_{j=1}^{d-1} \chi_{j}^{+}(\rho) \mathrm{d} \mu(\rho) .
$$

They also asked about the extension of this conjecture to manifolds without conjugate points [4]. In a recent work [16], we were able to prove that their conjecture holds for any surface with an Anosov geodesic flow (for instance surfaces of negative curvature). Regarding our proof and the nice properties of surfaces of nonpositive curvature [11,19], it became clear that our result can be adapted in the following way:

Theorem 1.1. Let $M$ be a compact, connected, $\mathcal{C}^{\infty}$ riemannian surface without boundary and of nonpositive sectional curvature. Let $\mu$ be a semiclassical measure. Then,

$$
h_{K S}(\mu, g) \geq \frac{1}{2} \int_{S^{*} M} \chi^{+}(\rho) \mathrm{d} \mu(\rho),
$$

where $h_{K S}(\mu, g)$ is the Kolmogorov-Sinai entropy and $\chi^{+}(\rho)$ is the upper Lyapunov exponent at point $\rho$.

In particular, this result shows that the support of any semiclassical measure cannot be reduced to closed unstable geodesics. We underline that our inequality is also coherent with the quasimodes constructed by Donnelly. In [9], he considered the question of Quantum Unique Ergodicity for packets of eigenfunctions and he proved that for this generalized question, you can construct exceptional sequences of quasimodes that concentrate on flat parts of the surface (even if the Liouville measure is ergodic) and that have in particular zero entropy. Our theorem on the entropy of semiclassical measures holds for sequences of eigenfunctions of the Laplacian. So the two situations are slightly different but our inequality on the entropy (if generalized to quasimodes) would be consistent with Donnelly's construction.

We can make a last observation on the assumptions on the manifold: it is not known whether the Liouville measure is ergodic or not for the geodesic flow on a surface of nonpositive curvature. In fact, if the genus of the surface is larger than 1, then the best known result in this direction is that there exists an open and dense invariant subset $U$ of positive Liouville measure such that the restriction $L_{\mid U}$ of the Liouville measure is ergodic with respect to $g_{\mid U}[6]$. The extension of this result on the entropy of semiclassical measures raises the 
question of knowing whether one could obtain an analogue of this result for weakly chaotic systems. For instance, regarding the construction from [14], it would be interesting to have a lower bound for ergodic billiards.

Our purpose in this article is to prove Theorem 1.1. Our strategy will be the same as in [16] (and also [4]) so it is probably better (and easier) for the reader to have a good understanding of the methods from these two references where the geometric situation is "simpler". We will focus on the main differences and refer the reader to $[4,16]$ for the details of several lemmas. The crucial observation is that as in the Anosov case, surfaces of nonpositive curvature have continuous stable and unstable foliations and no conjugate points. These properties were at the heart of the proofs in $[3,4,16]$ and we will verify that even if the properties of these stable/unstable directions are weaker for surfaces of nonpositive curvature, they are sufficient to answer the question of Anantharaman-Nonnenmacher in this weakly chaotic setting.

In $[3,4,16]$, there was a dynamical quantity which was crucially used: the unstable Jacobian of the geodesic flow. In the case of surfaces of nonpositive curvature, one can introduce an analogue of it. This quantity comes from the study of Jacobi fields and is called the unstable Riccati solution $U^{u}(\rho)$ [19]. In the case of surfaces without conjugate points, Freire and Mañé have shown that this quantity is related to the upper Lyapunov exponent at point $\rho$ [12]. In fact, for any $\left(g^{t}\right)_{t}$-invariant probability measure on $S^{*} M$, one has

$$
\mu \text { a.e., } \chi^{+}(\rho)=\lim _{T \rightarrow+\infty} \frac{1}{T} \int_{0}^{T} U^{u}\left(g^{s} \rho\right) \mathrm{d} s,
$$

where $\chi^{+}(\rho)$ is the upper Lyapunov exponent at point $\rho$. Thanks to the Birkhoff ergodic theorem, the Ruelle inequality can be then rewritten as follows:

$$
h_{K S}(\mu, g) \leq \int_{S^{*} M} U^{u}(\rho) \mathrm{d} \mu(\rho) .
$$

And also, the lower bound of Theorem 1.1 can be rewritten as

$$
h_{K S}(\mu, g) \geq \frac{1}{2} \int_{S^{*} M} U^{u}(\rho) \mathrm{d} \mu(\rho) .
$$

The main advantage of this new formulation is that the function in the integral of the lower bound is defined everywhere (and not almost everywhere).

Remark. One could also ask whether it would be possible to extend this result to surfaces without conjugate points. In fact, these surfaces also have a stable and unstable foliations with nice properties [19] (and of course no conjugate points). The main difficulty is that the continuity of $U^{u}(\rho)$ is not true anymore [5] and at this point, we do not see any way of escaping this difficulty. 


\subsection{Organization of the Article}

In Sect. 2, we will give a precise survey ${ }^{2}$ on surfaces of nonpositive curvature and highlight the properties we will need to make the proof works. As rewriting all the details of the proofs from $[4,16]$ would be very long and very similar to what was already done in these earlier works, we will refer the reader to them for the proofs of some lemmas and we will explain precisely which points need to be modified at the different stages of the argument. In Sect. 3, we will draw a precise outline of the proof. Then, in Sect. 4, we will explain how the main result from [4] can be adapted in the setting of surfaces of nonpositive curvature. In Sect. 5, we follow the same strategy as in [16] to derive a crucial estimate on the quantum pressures. Finally, in the appendix, we recall some results on quantum pressure from [4] and some facts about the KolmogorovSinai entropy.

\section{Classical Setting of the Article}

\subsection{Background on Surfaces of Nonpositive Curvature}

In this first section, we recall some facts about nonpositively curved manifolds [19] (chapter 3), [11]. From this point of the article, we fix $M$ to be a smooth, compact and connected riemannian surface of nonpositive sectional curvature which has no boundary.

2.1.1. Stable and Unstable Jacobi Fields. We define $\pi: S^{*} M \rightarrow M$ the canonical projection $\pi(x, \xi):=x$. The vertical subspace $V_{\rho}$ at $\rho=(x, \xi)$ is the kernel of the application $d_{\rho} \pi$. We underline that it is in fact the tangent space at $\rho$ of the 1-dimensional submanifold $S_{x}^{*} M$.

We can also define the horizontal subspace at $\rho$. Precisely, for $Z \in$ $T_{\rho} S^{*} M$, we consider a smooth curve $c(t)=(a(t), b(t)), t \in(-\epsilon, \epsilon)$, in $S^{*} M$ such that $c(0)=\rho$ and $c^{\prime}(0)=Z$. Then, we define the horizontal space $\mathcal{H}_{\rho}$ as the kernel of the application $\mathbb{K}_{\rho}(Z)=\nabla_{a^{\prime}(0)} b(0)=\nabla_{d_{\rho} \pi(Z)} b(0)$, where $\nabla$ is the Levi-Civita connection. This subspace contains $X_{H}(\rho)$ the vector field tangent to the Hamiltonian flow and it is of dimension 2.

We know that we can use these two subspaces to split the tangent space $T_{\rho} S^{*} M=\mathcal{H}_{\rho} \oplus V_{\rho}$. This allows us to define the Sasaki metric on $S^{*} M[19]$ (p. 18) that splits these two subspaces into orthogonal spaces, namely for every $\rho=(x, \xi) \in S^{*} M$ and for every $X, Y \in T_{\rho} S^{*} M$,

$$
\langle X, Y\rangle_{\rho}:=g_{x}\left(d_{\rho} \pi(X), d_{\rho} \pi(Y)\right)+g_{x}\left(\mathbb{K}_{\rho}(X), \mathbb{K}_{\rho}(Y)\right),
$$

where $g_{x}$ is the metric at $x$ on the riemannian manifold $M$. Using this decomposition, we would like to recall an important link between the linearization of the geodesic flow and the Jacobi fields on $M$. To do this, we underline that for each point $\rho$ in $S^{*} M$, there exists a unique unit speed geodesic $\gamma_{\rho}$. Then we define a Jacobi field in $\rho$ (or along $\gamma_{\rho}$ ) as a solution of the differential equation:

$$
\mathbb{J} "(t)+R\left(\gamma_{\rho}^{\prime}(t), \mathbb{J}(t)\right) \gamma_{\rho}^{\prime}(t)=0,
$$

\footnotetext{
${ }^{2}$ We refer the reader to $[11,19]$ for more details.
} 
where $R(X, Y) Z$ is the curvature tensor applied to the vector fields $X, Y$ and $Z$ and $\mathbb{J}^{\prime}(t)=\nabla_{\gamma_{\rho}^{\prime}(t)} \mathbb{J}(t)$.

Recall that we can interpret Jacobi fields as geodesic variation vector fields [11]. Precisely, consider a $\mathcal{C}^{\infty}$ family of curves $c_{s}:[a, b] \rightarrow M, s$ in $(-\epsilon, \epsilon)$. We say that it is a smooth variation of $c=c_{0}$. It defines a corresponding variation vector field $Y(t)=\frac{\partial}{\partial s}\left(c_{s}(t)\right)_{\mid s=0}$ that gives the initial velocity of $s \mapsto c_{s}(t)$. If we suppose now that $c$ is a geodesic of $M$, then a $\mathcal{C}^{2}$ vector field $Y(t)$ on $c$ is a Jacobi vector field if and only if $Y(t)$ is the variation vector field of a geodesic variation of $c$ (i.e. $\forall s \in(-\epsilon, \epsilon), c_{s}$ is a geodesic of $M$ ). For instance, $\gamma_{\rho}^{\prime}(t)$ and $t \gamma_{\rho}^{\prime}(t)$ are Jacobi vector fields along $\gamma_{\rho}$.

Consider now a vector $(V, W)$ in $T_{\rho} S^{*} M$ given in coordinates $\mathcal{H}_{\rho} \oplus$ $V_{\rho}$. Using the canonical identification given by $d_{\rho} \pi$ and $\mathbb{K}_{\rho}$, there exists a unique Jacobi field $\mathbb{J}_{V, W}(t)$ in $\rho$ whose initial conditions are $\mathbb{J}_{V, W}(0)=V$ and $\mathbb{J}_{V, W}^{\prime}(0)=W$, such that

$$
d_{\rho} g^{t}(V, W)=\left(\mathbb{J}_{V, W}(t), \mathbb{J}_{V, W}^{\prime}(t)\right)
$$

in coordinates $\mathcal{H}_{g^{t} \rho} \oplus V_{g^{t} \rho}\left[19\right.$, Lemma 1.4]. Define $N_{\rho}$ the subspace of $T_{\rho} S^{*} M$ of vectors orthogonal to $X_{H}(\rho)$ and $H_{\rho}$ the intersection of this subspace with $\mathcal{H}_{\rho}$. Using the previous property on Jacobi fields, we know that the subbundle $\mathcal{N}$ perpendicular to the Hamiltonian vector field is invariant by $g^{t}$ and that we have the following splitting [19] (Lemma 1.5):

$$
T_{\rho} S^{*} M=\mathbb{R} X_{H}(\rho) \oplus H_{\rho} \oplus V_{\rho} .
$$

These properties can be extended to any energy layer $\mathcal{E}(\lambda):=\left\{\|\xi\|_{x}^{2}=\lambda\right\}$ for any positive $\lambda$. Following [19, Lemma 3.1], we can construct two particular Jacobi fields along $\gamma_{\rho}$. We denote $\left(\gamma_{\rho}^{\prime}(t), e(t)\right)$ an orthonormal basis defined along $\gamma_{\rho}(t)$. Given a positive $T$ and because there are no conjugate points on the surface $M$ (Hadamard-Cartan theorem [11,19]), there exists a unique Jacobi field $\mathbb{J}_{T}(t)$ such that $\mathbb{J}_{T}(0)=e(0)$ and $\mathbb{J}_{T}(T)=0$. Moreover, $\mathbb{J}_{T}(t)$ is perpendicular to $\gamma_{\rho}(t)$ for all $t$ in $\mathbb{R}\left[19\right.$, p. 50]. As a consequence, $\mathbb{J}_{T}(t)$ can be identified with its coordinate along $e(t)$ ( as $T_{\gamma_{\rho}(t)} M$ is of dimension 2). A result due to Hopf (Lemma 3.1 in [19]) tells us that the limits

$$
\lim _{T \rightarrow+\infty} \mathbb{J}_{T}(t) \text { and } \lim _{T \rightarrow-\infty} \mathbb{J}_{T}(t)
$$

exist. They are denoted $\mathbb{J}_{\rho}^{s}(t)$ and $\mathbb{J}_{\rho}^{u}(t)$ (respectively the stable and the unstable Jacobi field). They satisfy the simplified one dimensional Jacobi equation:

$$
\mathbb{J}^{\prime \prime}(t)+K(t) \mathbb{J}(t)=0,
$$

where $K(t)=K\left(\gamma_{\rho}(t)\right)$ is the sectional curvature at $\gamma_{\rho}(t)$. They are never vanishing Jacobi fields with $\mathbb{J}_{\rho}^{*}(0)=e(0)$ and for all $t$ in $\mathbb{R}$, they are perpendicular to $\gamma_{\rho}^{\prime}(t)$. Moreover, we have $\left\|\mathbb{J}_{\rho}^{*^{\prime}}(t)\right\| \leq \sqrt{K_{0}}\left\|\mathbb{J}_{\rho}^{*}(t)\right\|$ for every $t$ in $\mathbb{R}$ (where $-K_{0}$ is some negative lower bound on the curvature).

Using the previous link between geodesic flow and Jacobi fields, we can lift these subspaces to invariant subspaces $E^{s}(\rho)$ and $E^{u}(\rho)$ called the Green stable and unstable subspaces. These subspaces have dimension 1 (as $M$ is a surface) and are included in $N_{\rho}$. A basis of $E^{s}\left(g^{t} \rho\right)$ is given by $\left(\mathbb{J}_{\rho}^{s}(t), \mathbb{J}_{\rho}^{s^{\prime}}(t)\right)$ in 
coordinates $H_{g^{t} \rho} \oplus V_{g^{t} \rho}$. We can underline that both subspaces are uniformly transverse to $V_{\rho}$ and that it can happen that they are equal to each other (which was not the case in the Anosov setting). In the case of nonpositive curvature, these subspaces depend continuously in $\rho$ and are integrable as in the Anosov case [11].

Remark. We underline that we could develop the same construction for manifolds without conjugate points and the same properties would be true except the continuity of the stable/unstable foliation [5].

In the case where the Green subspaces attached to $\rho$ are linearly independent, a splitting of $N_{\rho}$ is given by $E^{u}(\rho) \oplus E^{s}(\rho)$ and the splitting holds for all the trajectory. For the opposite case, we know that the Green subspaces attached to $\rho$ (and hence to a geodesic $\gamma_{\rho}$ ) are linearly dependent if and only if the sectional curvature is vanishing at every point of the geodesic $\gamma_{\rho}$ [19]. As a consequence, we cannot use the same kind of splitting. However, there exists a splitting of $N_{\rho}$ that we can use in both cases, precisely $E^{u}(\rho) \oplus V_{\rho}$.

2.1.2. Riccati Equation. The one dimensional Jacobi equation defined earlier gives rise to the Riccati equation:

$$
U^{\prime}(t)+U^{2}(t)+K(t)=0
$$

where $U(t)=\mathbb{J}^{\prime}(t) \mathbb{J}(t)^{-1}$ for non vanishing $\mathbb{J}$. Then, we define the corresponding unstable Riccati solution associated to the unstable Jacobi field as $U_{\rho}^{u}(t):=$ $\mathbb{J}_{\rho}^{u^{\prime}}(t)\left(\mathbb{J}_{\rho}^{u}(t)\right)^{-1}$. It is a nonnegative quantity that controls the growth of the unstable Jacobi field (in dimension 2) as follows:

$$
\left\|\rrbracket_{\rho}^{u}(t)\right\|=\left\|\rrbracket_{\rho}^{u}(0)\right\| e^{\int_{0}^{t} U_{\rho}^{u}(s) \mathrm{d} s} .
$$

The same works for the stable Jacobi field. Both quantities are continuous ${ }^{3}$ with respect to $\rho$. We underline that, we can use the previous results to obtain the bound $\left\|d_{\rho} g_{\mid E^{u}(\rho)}^{t}\right\| \leq \sqrt{1+K_{0}} e^{\int_{0}^{t} U_{\rho}^{u}(s) \mathrm{d} s}[19$, pp. 53-54]. So the unstable Riccati solution describe the infinitesimal growth of the geodesic flow along the unstable direction. As for the unstable Jacobian, Freire and Mañé showed that the unstable Riccati solutions are related to the Lyapunov exponents [12]. In fact, they proved that in the case of nonpositive curvature (and more generally for surfaces without conjugate points), the upper Lyapunov exponent at point $\rho$ of a $\left(g^{t}\right)_{t}$-invariant measure $\mu$ is given by

$$
\mu \text { a.e., } \chi^{+}(\rho)=\lim _{T \rightarrow+\infty} \frac{1}{T} \int_{0}^{T} U^{u}\left(g^{s} \rho\right) \mathrm{d} s .
$$

\footnotetext{
3 The continuity in $\rho$ is a crucial property that we will use in our proof. We underline that it is not true if we only suppose the surface to be without conjugate points [5].
} 
2.1.3. Divergence of Vanishing Jacobi Fields. A last point we would like to recall is a result due to Green [13] and to Eberlein in the general case [10]. It asserts that for any positive $c$ there exists a positive $T=T(c)$ such that for any $\rho$ in $S^{*} M$ and for any nontrivial Jacobi field $\mathbb{J}(t)$ along $\gamma_{\rho}$ such that $\mathbb{J}(0)=0$ and $\left\|\mathbb{J}^{\prime}(0)\right\| \geq 1$, for all $t$ larger than $T$, we have $\|\mathbb{J}(t)\| \geq c$ [19, Proposition 3.1]). In the case of manifolds without conjugate points, this property of uniform divergence only holds in dimension 2 and it will be crucially used in the following (for manifolds without conjugate points of higher dimension, the same result holds but without any uniformity in $\rho$ ). Finally, all these properties allow to prove the following lemma:

Lemma 2.1. Let $v=(0, V)$ be a unit vertical vector at $\rho$. Then for any $c>0$, there exists $T=T(c)>0$ (independent of $\rho$ and of $v$ ) such that for any $t \geq T$, $\left\|d_{\rho} g^{t} v\right\| \geq c$.

As $g^{t}$ preserves the riemannian volume on $S^{*} M$ (given by the Sasaki metric), we know that the jacobian of $d_{\rho} g^{t}$ from $N_{\rho}=E^{u}(\rho) \oplus V_{\rho}$ to $N_{g^{t} \rho}=$ $E^{u}\left(g^{t} \rho\right) \oplus V_{g^{t} \rho}$ is uniformly bounded. Combining the fact $\left\|d_{\rho} g^{t} v^{u}\right\|$ is nondecreasing for every $v^{u}$ in $E^{u}(\rho)$ (and every $t \geq 0$ ) and Lemma 2.1, we find that, for $\kappa>0$, there exists $T=T(\kappa)$ such that the angle between $E^{u}\left(g^{t} \rho\right)$ and $\mathbb{R} d_{\rho} g^{t} v$ is bounded by some $\kappa$ for every $t \geq T$, for every $\rho$ in $S^{*} M$ and for every unit vector $v$ in $V_{\rho}$.

As, it will be useful in the article, we would like to show that our discussion allows to have a control of $\left\|d_{\rho} g^{t}\right\|$ (for $t \geq 0$ ) in terms of the unstable Riccati solution.

In order to obtain this control, we use the splitting of $T_{\rho} S^{*} M$ given by $\mathbb{R} X_{H}(\rho) \oplus E^{u}(\rho) \oplus V_{\rho}$. These three subspaces are uniformly transverse so we only have to give an estimate of $\left\|d_{\rho} g_{E \rightarrow T_{g^{t}}^{*}}^{t} M\right\|$ when $E$ is one of them. In the case where $E=\mathbb{R} X_{H}(\rho)$, it is bounded by 1 and in the case where $E=E^{u}(\rho)$, it is bounded by $\sqrt{1+K_{0}} e^{\int_{0}^{t} U_{\rho}^{u}(s) \mathrm{d} s}$. In the last case, we fix a small step of time $\eta>0$. Then, we consider $e_{0}$ a unit vector in $V_{\rho}$ and for $0 \leq p \eta \leq t$, we define the $e_{p \eta}$ as the unit vector $\frac{d_{\rho} g^{p \eta} e_{0}}{\left\|d_{\rho} g^{p \eta} e_{0}\right\|}$. We can write, for $k:=[t / \eta]$,

$$
\begin{aligned}
\left\|d_{\rho} g^{k \eta} e_{0}\right\|_{g^{k \eta} \rho} & =\left|\left\langle d_{\rho} g^{k \eta} e_{0}, e_{k \eta}\right\rangle_{g^{k \eta} \rho}\right| \\
& =\left|\left\langle d_{g^{(k-1) \eta} \rho} g^{\eta} e_{(k-1) \eta}, e_{k \eta}\right\rangle_{g^{k \eta} \rho} \cdots\left\langle d_{\rho} g^{\eta} e_{0}, e_{\eta}\right\rangle_{g^{\eta} \rho}\right| .
\end{aligned}
$$

We also define the corresponding sequence $e_{p \eta}^{u}:=\frac{d_{\rho} g^{p \eta} e_{0}^{u}}{\left\|d_{\rho} g^{p \eta} e_{0}^{u}\right\|}$ of unit unstable vectors, where $e_{0}^{u}:=\frac{\left(J_{\rho}^{u}(0), J_{\rho}^{u^{\prime}}(0)\right)}{\left\|\left(J_{\rho}^{u}(0), J_{\rho}^{u^{\prime}}(0)\right)\right\|_{\rho}}$. From Lemma 2.1, we know that $e_{p \eta}$ becomes uniformly close (in $\rho$ ) to $e_{p \eta}^{u}$. So, $\log \left|\left\langle d_{g^{(p-1) \eta} \rho} g^{\eta} e_{(p-1) \eta}, e_{p \eta}\right\rangle_{g^{p \eta} \rho}\right|$ becomes uniformly close to $\log \left|\left\langle d_{g^{(p-1) \eta} \rho} g^{\eta} e_{(p-1) \eta}^{u}, e_{p \eta}^{u}\right\rangle_{g^{p \eta} \rho}\right|$. In particular, for every $\delta^{\prime}>0$, there exists a constant $C>0$ such that

$$
\left\|d_{\rho} g^{k \eta} e_{0}\right\|_{g^{k \eta} \rho} \leq C e^{k \eta \delta^{\prime}}\left|\left\langle d_{g^{(k-1) \eta} \rho} g^{\eta} e_{(k-1) \eta}^{u}, e_{k \eta}^{u}\right\rangle_{g^{k \eta} \rho} \cdots\left\langle d_{\rho} g^{\eta} e_{0}^{u}, e_{\eta}^{u}\right\rangle_{g^{\eta} \rho}\right| .
$$

Again, this last quantity is equal to $C e^{k \eta \delta^{\prime}}\left\|d_{\rho} g^{k \eta} e_{0}^{u}\right\|_{g^{k \eta} \rho}$. From the properties of the unstable Riccati solution, this quantity is bounded by $C e^{k \eta \delta^{\prime}} e^{\int_{0}^{k \eta} U_{\rho}^{u}(s) \mathrm{d} s}$ 
(with $C$ uniform in $\rho$ ). As the subspaces $\mathbb{R} X_{H}(\rho), V_{\rho}$ and $E^{u}(\rho)$ are uniformly transverse to each other, we finally deduce that for every $\delta^{\prime}>0$, there exists $C>0$ such that for every $\rho \in S^{*} M,\left\|d_{\rho} g^{t}\right\| \leq C e^{t \delta^{\prime}} e^{\int_{0}^{t} U_{\rho}^{u}(s) \mathrm{d} s}$.

\subsection{Discretization of the Unstable Riccati Solution}

For $\theta$ small positive number ( $\theta$ will be fixed all along the paper), one defines

$$
\mathcal{E}^{\theta}:=\left\{(x, \xi) \in T^{*} M: 1-2 \theta \leq\|\xi\|_{x}^{2} \leq 1+2 \theta\right\} .
$$

From previous section, we know that there exists a constant $b_{0}$ such that

$$
\forall \rho \in \mathcal{E}^{\theta}, \quad 0 \leq U^{u}(\rho) \leq b_{0} .
$$

This function will replace the logarithm of the unstable Jacobian $\log J^{u}$ in the proof from [16]. The situation is slightly different from the case of an Anosov flow as we do not have that $U^{u}$ is uniformly bounded from below by some positive constant, a property that was crucially to prove Theorem 1.2 in [16]. We solve this problem by introducing a small positive parameter $\epsilon_{0}$ and defining an auxiliary function

$$
U_{0}^{u}(\rho):=\sup \left\{U^{u}(\rho), \epsilon_{0}\right\} .
$$

We also fix $\epsilon$ and $\eta$ two small positive constants lower than the injectivity radius of the manifold (that we suppose to be larger than 2 ). We choose $\eta$ small enough to have $\left(2+\frac{b_{0}}{\epsilon_{0}}\right) b_{0} \eta \leq \frac{\epsilon}{2}$ (as in [16], this property is only used in the proof of Lemma 3.1). We underline that there exists $d_{0}>0$ such that if

$$
\forall\left(\rho, \rho^{\prime}\right) \in \mathcal{E}^{\theta} \times \mathcal{E}^{\theta}, \quad d\left(\rho, \rho^{\prime}\right) \leq d_{0} \Rightarrow\left|U^{u}(\rho)-U^{u}\left(\rho^{\prime}\right)\right| \leq \epsilon_{0} \epsilon .
$$

We also choose $\eta$ small enough to have

$$
\forall \rho \in \mathcal{E}^{\theta}, \quad \forall 0 \leq s \leq \eta, \quad\left|U^{u}(\rho)-U^{u}\left(g^{s} \rho\right)\right| \leq \epsilon \epsilon_{0} .
$$

We make the extra assumption that the small parameter $\epsilon$ used for the continuity is smaller than $\epsilon_{0}$, i.e.

$$
\left(2+\frac{b_{0}}{\epsilon_{0}}\right) b_{0} \eta \leq \frac{\epsilon}{2} \ll \epsilon_{0} .
$$

In particular, $d_{0}$ can (and will) be chosen independently of $\epsilon_{0}$ (by taking $\epsilon^{2} / 2$ instead of $\epsilon \epsilon_{0}$ in the previous continuity relations).

Discretization of the Manifold. As in the case of Anosov surfaces, our strategy to prove Theorem 1.1 will be to introduce a discrete reparametrization of the geodesic flow. Regarding this goal, we cut the manifold $M$ and precisely, we consider a partition $M=\bigsqcup_{i=1}^{K} O_{i}$ of diameter smaller than some positive $\delta$. Let $\left(\Omega_{i}\right)_{i=1}^{K}$ be a finite open cover of $M$ such that for all $1 \leq i \leq K, O_{i} \Subset \Omega_{i}$. For $\gamma \in\{1, \ldots, K\}^{2}$, define the following open subset of $T^{*} M$ :

$$
V_{\gamma}:=\left(T^{*} \Omega_{\gamma_{0}} \cap g^{-\eta} T^{*} \Omega_{\gamma_{1}}\right) \cap \mathcal{E}^{\theta} .
$$

We choose the partition $\left(O_{i}\right)_{i=1}^{K}$ and the open cover $\left(\Omega_{i}\right)_{i=1}^{K}$ of $M$ such that $\left(V_{\gamma}\right)_{\gamma \in\{1, \ldots, K\}^{2}}$ is a finite open cover of diameter smaller ${ }^{4}$ than $d_{0}$ of $\mathcal{E}^{\theta}$. For

\footnotetext{
${ }^{4}$ In particular, the diameter of the partition depends on $\theta$ and $\epsilon$ (but not on $\epsilon_{0}$ ).
} 
$\gamma:=\left(\gamma_{0}, \gamma_{1}\right)$, we define $f(\gamma)$ and $f_{0}(\gamma)$ as in the case of an Anosov flow i.e.

$$
f_{0}(\gamma):=\eta \inf \left\{U_{0}^{u}(\rho): \rho \in V_{\gamma}\right\} \quad \text { and } \quad f(\gamma):=\eta \inf \left\{U^{u}(\rho): \rho \in V_{\gamma}\right\},
$$

if $V_{\gamma}$ is nonempty, $\eta b_{0}$ otherwise. Compared with the Anosov case, we will have slightly different properties for the function $f(\gamma)$, i.e.

$$
\forall \rho \in V_{\gamma}, \quad\left|\int_{0}^{\eta} U_{\rho}^{u}(s) \mathrm{d} s-f(\gamma)\right| \leq 2 \eta \epsilon_{0} \epsilon .
$$

We also underline that the function $f_{0}$ satisfies the following bounds, for $\gamma \in$ $\{1, \ldots, K\}^{2}$,

$$
\epsilon_{0} \eta \leq f_{0}(\gamma) \leq b_{0} \eta
$$

Finally, let $\alpha=\left(\alpha_{0}, \alpha_{1}, \ldots\right)$ be a (finite or infinite) sequence of elements in $\{1, \ldots, K\}$ whose length is larger than 1 and define:

$$
f_{+}(\alpha):=f_{0}\left(\alpha_{0}, \alpha_{1}\right) \leq \frac{\epsilon}{2} \quad \text { and } \quad f(\alpha):=f\left(\alpha_{0}, \alpha_{1}\right) \leq \frac{\epsilon}{2} .
$$

In the following, we will also have to consider negative times. To do this, we define the analogous functions, for $\beta:=\left(\ldots, \beta_{-1}, \beta_{0}\right)$ of finite (or infinite) length,

$$
f_{-}(\beta):=f_{0}\left(\beta_{-1}, \beta_{0}\right) \text { and } f(\beta):=f\left(\beta_{-1}, \beta_{0}\right) .
$$

Remark. We underline that the functions $f_{+}$and $f_{-}$are defined from $U_{0}^{u}$ while $f$ is defined from $U^{u}$. This distinction will be important in the following.

\section{Proof of Theorem 1.1}

Let $\left(\psi_{\hbar_{k}}\right)$ be a sequence of orthonormal eigenfunctions of the Laplacian corresponding to the eigenvalues $-\hbar_{k}^{-2}$ such that the corresponding sequence of distributions $\mu_{k}$ on $T^{*} M$ converges as $k$ tends to infinity to the semiclassical measure $\mu$. For simplicity of notations and to fit semiclassical analysis notations, we will denote $\hbar$ tends to 0 the fact that $k$ tends to infinity and $\psi_{\hbar}$ and $-\hbar^{-2}$ the corresponding eigenvector and eigenvalue. To prove the inequality of Theorem 1.1, we will give a symbolic interpretation of a semiclassical measure and apply results on suspension flows to this measure [1].

Let $\epsilon^{\prime}>4 \epsilon$ be a positive number, where $\epsilon$ was defined in Sect. 2.2. As in the Anosov setting, the link between the two quantities $\epsilon$ and $\epsilon^{\prime}$ is only used to obtain a theorem on product of pseudodifferential operators from Sects. 6 and 7 in [16] (here Theorem 3.2). In the following of the article, the Ehrenfest time $n_{E}(\hbar)$ will be the quantity

$$
n_{E}(\hbar):=\left[\left(1-\epsilon^{\prime}\right)|\log \hbar|\right] .
$$

We underline that it is an integer time and that, compared with usual definitions of the Ehrenfest time, there is no dependence on the Lyapunov exponent. We also consider a smaller non integer time

$$
T_{E}(\hbar):=(1-\epsilon) n_{E}(\hbar) .
$$


Before entering the details of the proof, we would like to say a few words about the Ehrenfest time and about ideas that are behind our strategy from [16]. In order to prove Theorem 1.1, we need to have a precise understanding of the range of validity of the semiclassical approximation. For instance, if one considers a smooth symbol $a$ compactly supported in a small neighborhood of $S^{*} M$, it can be shown [4] that

$$
\forall|t| \leq \frac{T_{E}(\hbar)}{2 \lambda_{\max }}, \quad\left\|e^{-\frac{\imath t \hbar \Delta}{2}} \mathrm{Op}_{\hbar}(a) e^{\frac{\imath t \hbar \Delta}{2}}-\mathrm{Op}_{\hbar}\left(a \circ g^{t}\right)\right\|_{L^{2}(M) \rightarrow L^{2}(M)}=o_{a}(1)
$$

where $\lambda_{\max }:=\lim _{t \rightarrow \pm \infty} \frac{1}{t} \log \sup _{\rho \in S^{*} M}\left|d_{\rho} g^{t}\right|$ is the maximal expansion rate of the geodesic flow. This result tells us that the semiclassical approximation remains valid for times of order $T_{E}(\hbar) /\left(2 \lambda_{\max }\right)$. This was the version of the Egorov theorem that was used by Anantharaman, Koch and Nonnenmacher in $[3,4]$ and the $\lambda_{\max }$ term in their lower bound came from this Egorov property. In [16], we managed to overcome this problem by observing that the range of validity of the semiclassical approximation depends also on the symbol you consider. In order to compute entropy, the symbols we will be interested in will in fact be of the form $Q_{\alpha_{0}} \times \cdots Q_{\alpha_{k}} \circ g^{k \eta}$ where $Q_{\alpha_{j}}$ is compactly supported in $T^{*} \Omega_{\alpha_{j}} \cap \mathcal{E}^{\theta}$ (see (41) for instance). An important aspect of the proof is that this symbol remains in a nice class of symbols amenable to pseudodifferential calculus as long as

$$
\sum_{j=0}^{k-2} f_{0}\left(\alpha_{j}, \alpha_{j+1}\right) \leq \frac{T_{E}(\hbar)}{2}
$$

An analogous property was used in [16, Sect. 7] in order to prove a subadditivity property (here Theorem 3.2). This property means that there exists a local time for which the range of validity of the semiclassical approximation is longer than the usual Ehrenfest time $T_{E}(\hbar) / \lambda_{\max }$. Precisely, the largest integer $k$ for which relation (10) is true will be the local Ehrenfest time for the symbol $Q_{\alpha_{0}} \times \cdots Q_{\alpha_{k}} \circ g^{k \eta}$. In order to prove our main theorem, we will introduce a "suspension of the quantum dynamics" for which the sum in (10) will appear naturally.

We draw now a precise outline of the proof of Theorem 1.1 which is similar to the one we used in the Anosov case [16]. We will refer the reader to this reference for the proof of several lemmas. The main differences with the Anosov case is that we have to introduce a thermodynamical formalism to treat the problem of flat parts of the surface.

\subsection{Quantum Partitions of Identity}

In order to find a lower bound on the metric entropy of the semiclassical measure $\mu$, we would like to apply the uncertainty principle for quantum pressure (see Appendix A) and see what informations it will give (when $\hbar$ tends to 0) on the metric entropy of the semiclassical measure $\mu$. To do this, we define quantum partitions of identity corresponding to a given partition of the manifold. 
3.1.1. Partitions of Identity. In Sect. 2.2, we considered a partition of small diameter $\left(O_{i}\right)_{i=1}^{K}$ of $M$. We also defined $\left(\Omega_{i}\right)_{i=1}^{K}$ a corresponding finite open cover of small diameter of $M$. By convolution of the characteristic functions $\mathbf{1}_{O_{i}}$, we obtain $\mathcal{P}=\left(P_{i}\right)_{i=1, \ldots, K}$ a smooth partition of unity on $M$, i.e. for all $x \in M$

$$
\sum_{i=1}^{K} P_{i}^{2}(x)=1 .
$$

We assume that for all $1 \leq i \leq K, P_{i}$ is an element of $\mathcal{C}_{c}^{\infty}\left(\Omega_{i}\right)$. To this classical partition corresponds a quantum partition of identity of $L^{2}(M)$. In fact, if $P_{i}$ denotes the multiplication operator by $P_{i}(x)$ on $L^{2}(M)$, then one has:

$$
\sum_{i=1}^{K} P_{i}^{*} P_{i}=\operatorname{Id}_{L^{2}(M)} .
$$

\subsubsection{Refinement of the Quantum Partition Under the Schrödinger Flow.} Like in the classical setting of entropy, we would like to make a refinement of the quantum partition. To do this refinement, we use the Schrödinger propagation operator $U^{t}=e^{\frac{\imath t \hbar \Delta}{2}}$. We define $A(t):=U^{-t} A U^{t}$, where $A$ is an operator on $L^{2}(M)$. To fit as much as possible with the metric entropy, we define the following operators:

$$
\tau_{\alpha}=P_{\alpha_{k}}(k \eta) \cdots P_{\alpha_{1}}(\eta) P_{\alpha_{0}}
$$

and

$$
\pi_{\beta}=P_{\beta_{-k}}(-k \eta) \cdots P_{\beta_{-2}}(-2 \eta) P_{\beta_{0}} P_{\beta_{-1}}(-\eta),
$$

where $\alpha=\left(\alpha_{0}, \ldots, \alpha_{k}\right)$ and $\beta=\left(\beta_{-k}, \ldots, \beta_{0}\right)$ are finite sequences of symbols such that $\alpha_{j} \in[1, K]$ and $\beta_{-j} \in[1, K]$. We can remark that the definition of $\pi_{\beta}$ is the analogue for negative times of the definition of $\tau_{\alpha}$. The only difference is that we switch the two first terms $\beta_{0}$ and $\beta_{-1}$. The reason of this choice relies on the application of the quantum uncertainty principle (see Appendix A). One can see that for fixed $k$ and using rules of pseudodifferential calculus,

$$
\left\|P_{\alpha_{k}}(k \eta) \cdots P_{\alpha_{1}}(\eta) P_{\alpha_{0}} \psi_{\hbar}\right\|^{2} \rightarrow \mu\left(P_{\alpha_{k}}^{2} \circ g^{k \eta} \times \cdots P_{\alpha_{1}}^{2} \circ g^{\eta} \times P_{\alpha_{0}}^{2}\right) \text { as } \hbar \rightarrow 0 .
$$

This last quantity is the one used to compute $h_{K S}\left(\mu, g^{\eta}\right)$ (with the notable difference that the $P_{j}$ are here smooth functions instead of characteristic functions). As in [16], we will study for which range of times, the operator $\tau_{\alpha}$ is a pseudodifferential operator of symbol $P_{\alpha_{k}} \circ g^{k \eta} \times \cdots P_{\alpha_{1}} \circ g^{\eta} \times$ $P_{\alpha_{0}}$. In [4] and [3], they only considered $k \eta \leq|\log \hbar| / \lambda_{\max }$ where $\lambda_{\max }:=$ $\lim _{t \rightarrow \pm \infty} \frac{1}{t} \log \sup _{\rho \in S^{*} M}\left|d_{\rho} g^{t}\right|$. This choice was not optimal and in the following, we will define sequences $\alpha$ for which we can say that $\tau_{\alpha}$ is a pseudodifferential operator. 


\subsubsection{Index Family Adapted to the Variation of the Unstable Riccati Solution.} Let $\alpha=\left(\alpha_{0}, \alpha_{1}, \ldots\right)$ be a (finite or infinite) sequence of elements of $\{1, \ldots, K\}$ whose length is larger than 1 . We define a natural shift on these sequences

$$
\sigma_{+}\left(\left(\alpha_{0}, \alpha_{1}, \ldots\right)\right):=\left(\alpha_{1}, \ldots\right) .
$$

For negative times and for $\beta:=\left(\ldots, \beta_{-1}, \beta_{0}\right)$, we define the backward shift

$$
\sigma_{-}\left(\left(\ldots, \beta_{-1}, \beta_{0}\right)\right):=\left(\ldots, \beta_{-1}\right) .
$$

In the following, we will mostly use the symbol $x$ for infinite sequences and reserve $\alpha$ and $\beta$ for finite ones. Then, using notations of Sect. 2.1, index families depending on the value of the unstable Riccati solutions can be defined as follows:

$$
\begin{aligned}
I^{\eta}(\hbar):=\left\{\left(\alpha_{0}, \ldots, \alpha_{k}\right): \sum_{i=1}^{k-2} f_{+}\left(\sigma_{+}^{i} \alpha\right) \leq T_{E}(\hbar)<\sum_{i=1}^{k-1} f_{+}\left(\sigma_{+}^{i} \alpha\right)\right\}, \\
K^{\eta}(\hbar):=\left\{\left(\beta_{-k}, \ldots, \beta_{0}\right): \sum_{i=1}^{k-2} f_{-}\left(\sigma_{-}^{i} \beta\right) \leq T_{E}(\hbar)<\sum_{i=1}^{k-1} f_{-}\left(\sigma_{-}^{i} \beta\right)\right\} .
\end{aligned}
$$

We underline that $f_{+}, f_{-} \geq \epsilon_{0} \eta$ ensures that we consider finite sequences. These sets define the maximal sequences for which we can expect rules from symbolic calculus to hold for the corresponding $\tau_{\alpha}$. The sums used to define these sets were already used in [16]. We can think of the time $|\alpha| \eta$ as a stopping time for which $\tau_{\alpha}$ remains a nice pseudodifferential operator in a nice class of symbols.

A good way of thinking of these families of words is by introducing the sets

$$
\Sigma_{+}:=\{1, \ldots, K\}^{\mathbb{N}} \text { and } \quad \Sigma_{-}:=\{1, \ldots, K\}^{-\mathbb{N}} .
$$

Once more, the sets $I^{\eta}(\hbar)$ (resp. $K^{\eta}(\hbar)$ ) lead to natural partitions of $\Sigma_{+}$ (resp. $\Sigma_{-}$). Families of operators can be associated to these families of index: $\left(\tau_{\alpha}\right)_{\alpha \in I^{\eta}(\hbar)}$ and $\left(\pi_{\beta}\right)_{\beta \in K^{\eta}(\hbar)}$. One can show that these partitions form quantum partitions of identity (Lemma 5.1 in [16]):

$$
\sum_{\alpha \in I^{\eta}(\hbar)} \tau_{\alpha}^{*} \tau_{\alpha}=\operatorname{Id}_{L^{2}(M)} \quad \text { and } \sum_{\beta \in K^{\eta}(\hbar)} \pi_{\beta}^{*} \pi_{\beta}=\operatorname{Id}_{L^{2}(M)} .
$$

\subsection{Symbolic Interpretation of Semiclassical Measures}

Now that we have defined these partitions of variable size, we want to show that they are adapted to compute the pressure of a certain measure with respect to some reparametrized flow associated to the geodesic flow. To do this, we provide a symbolic interpretation of the quantum partitions. We denote $\Sigma_{+}:=\{1, \ldots, K\}^{\mathbb{N}}$. We also denote $\mathcal{C}_{i}$ the subset of sequences $\left(x_{n}\right)_{n \in \mathbb{N}}$ such that $x_{0}=i$. Define also:

$$
\left[\alpha_{0}, \ldots, \alpha_{k}\right]:=\mathcal{C}_{\alpha_{0}} \cap \cdots \cap \sigma_{+}^{-k} \mathcal{C}_{\alpha_{k}},
$$


where $\sigma_{+}$is the shift $\sigma_{+}\left(\left(x_{n}\right)_{n \in \mathbb{N}}\right)=\left(x_{n+1}\right)_{n \in \mathbb{N}}$ (it fits the notations of the previous section). The set $\Sigma_{+}$is then endowed with the probability measure (not necessarily $\sigma_{+}$-invariant):

$$
\mu_{\hbar}^{\Sigma_{+}}\left(\left[\alpha_{0}, \ldots, \alpha_{k}\right]\right)=\mu_{\hbar}^{\Sigma_{+}}\left(\mathcal{C}_{\alpha_{0}} \cap \cdots \cap \sigma_{+}^{-k} \mathcal{C}_{\alpha_{k}}\right)=\left\|P_{\alpha_{k}}(k \eta) \cdots P_{\alpha_{0}} \psi_{\hbar}\right\|^{2} .
$$

Using the property of partition of identity, it is clear that this definition ensures the compatibility conditions to define a probability measure [22]:

$$
\sum_{\alpha_{k+1}} \mu_{\hbar}^{\Sigma_{+}}\left(\left[\alpha_{0}, \ldots, \alpha_{k+1}\right]\right)=\mu_{\hbar}^{\Sigma_{+}}\left(\left[\alpha_{0}, \ldots, \alpha_{k}\right]\right) .
$$

Then, we can define a suspension flow, in the sense of Abramov, associated to this probability measure. To do this, the suspension set is defined as

$$
\bar{\Sigma}_{+}:=\left\{(x, s) \in \Sigma_{+} \times \mathbb{R}_{+}: 0 \leq s<f_{+}(x)\right\} .
$$

Recall that the roof function $f_{+}$is defined as $f_{+}(x):=f_{0}\left(x_{0}, x_{1}\right)$. We define a probability measure $\bar{\mu}_{\hbar}^{\bar{\Sigma}_{+}}$on $\bar{\Sigma}_{+}$:

$$
\bar{\mu}_{\hbar}^{\bar{\Sigma}_{+}}=\frac{\mu_{\hbar}^{\Sigma_{+}} \times d t}{\int_{\Sigma_{+}} f_{+} \mathrm{d} \mu_{\hbar}^{\Sigma_{+}}} .
$$

The suspension semi-flow associated to $\sigma_{+}$is for time $s$ :

$$
\bar{\sigma}_{+}^{s}(x, t):=\left(\sigma_{+}^{n-1}(x), s+t-\sum_{j=0}^{n-2} f_{+}\left(\sigma_{+}^{j} x\right)\right),
$$

where $n$ is the only integer such that $\sum_{j=0}^{n-2} f_{+}\left(\sigma_{+}^{j} x\right) \leq s+t<\sum_{j=0}^{n-1} f_{+}\left(\sigma_{+}^{j} x\right)$.

Remark. We underline that we used the fact that $f_{+}>0$ to define the suspension flow. If we had considered $f$, we would not have been able to construct the suspension flow as $f$ could be equal to 0 .

Remark. It can be underlined that the same procedure holds for the partition $\left(\pi_{\beta}\right)$. The only differences are that we have to consider $\Sigma_{-}:=\{1, \ldots, K\}^{-\mathbb{N}}$, $\sigma_{-}\left(\left(x_{n}\right)_{n \leq 0}\right)=\left(x_{n-1}\right)_{n \leq 0}$ and that the corresponding measure is, for $k \geq 1$ :

$$
\begin{aligned}
\mu_{\hbar}^{\Sigma_{-}}\left(\left[\beta_{-k}, \ldots, \beta_{0}\right]\right) & =\mu_{\hbar}^{\Sigma_{-}}\left(\sigma_{-}^{-k} \mathcal{C}_{\beta_{-k}} \cap \cdots \cap \mathcal{C}_{\beta_{0}}\right) \\
& =\left\|P_{\beta_{-k}}(-k \eta) \cdots P_{\beta_{0}} P_{\beta_{-1}}(-\eta) \psi_{\hbar}\right\|^{2} .
\end{aligned}
$$

For $k=0$, one should take the only possibility to assure the compatibility condition:

$$
\mu_{\hbar}^{\Sigma_{-}}\left(\left[\beta_{0}\right]\right)=\sum_{j=1}^{K} \mu_{\hbar}^{\Sigma_{-}}\left(\left[\beta_{-1}, \beta_{0}\right]\right) .
$$

The definition is quite different from the positive case but in the semiclassical limit, it will not change anything as $P_{\beta_{0}}$ and $P_{\beta_{-1}}(-\eta)$ commute. Finally, the "past" suspension set can be defined as

$$
\bar{\Sigma}_{-}:=\left\{(x, s) \in \Sigma_{-} \times \mathbb{R}_{+}: 0 \leq s<f_{-}(x)\right\} .
$$


Consider the partition $\tilde{\mathcal{C}}:=([\alpha])_{\alpha \in I^{\eta}(\hbar)}$ of $\Sigma_{+}$. A partition $\overline{\mathcal{C}}_{\hbar}^{+}$of $\bar{\Sigma}_{+}$ can be defined starting from the partition $\tilde{\mathcal{C}}$ and $\left[0, f_{+}(\alpha)[\right.$. An atom of this suspension partition is an element of the form $\overline{\mathcal{C}}_{\alpha}^{+}=[\alpha] \times\left[0, f_{+}(\alpha)\left[\right.\right.$. For $\bar{\Sigma}^{-}$ (the suspension set corresponding to $\Sigma_{-}$), we define an analogous partition $\overline{\mathcal{C}}_{\hbar}^{-}=\left([\beta] \times\left[0, f_{-}(\beta)[)_{\beta \in K^{\eta}(\hbar)}\right.\right.$.

In the case of the Anosov geodesic flows [16], we used these partitions and show that they could be used to interpret some quantum entropy as the entropy of a refined partition of $\bar{\Sigma}^{+}$. Then, we used an entropic uncertainty principle taken from [4] to derive a lower bound on the quantum entropy. We will do the same thing here but we will have to be more careful and we will apply the entropic uncertainty principle for quantum pressures as in [4] (see Sect. A for a brief reminder). We introduce the weights

$$
W_{\alpha}^{+}:=\exp \left(\frac{1}{2} \sum_{j=1}^{k-1} f\left(\sigma_{+}^{j} \alpha\right)\right) \quad \text { and } \quad W_{\beta}^{-}:=\exp \left(\frac{1}{2} \sum_{j=1}^{k-1} f\left(\sigma_{-}^{j} \beta\right)\right) .
$$

We underline that the weights depends on $f$ and not on $f_{+}$or $f_{-}$. It came from the fact that $f$ is the function that appears in Theorem 4.1. We introduce the associated quantum pressures ${ }^{5}$

$$
p\left(\bar{\mu}_{\hbar}^{\bar{\Sigma}_{+}}, \overline{\mathcal{C}}_{\hbar}^{+}\right):=H\left(\bar{\mu}_{\hbar}^{\bar{\Sigma}_{+}}, \overline{\mathcal{C}}_{\hbar}^{+}\right)-2 \sum_{\alpha \in I^{\eta}(\hbar)} \bar{\mu}_{\hbar}^{\bar{\Sigma}_{+}}\left(\overline{\mathcal{C}}_{\alpha}^{+}\right) \log W_{\alpha}^{+}
$$

and

$$
p\left(\bar{\mu}_{\hbar}^{\bar{\Sigma}_{-}}, \overline{\mathcal{C}}_{\hbar}^{-}\right):=H\left(\bar{\mu}_{\hbar}^{\bar{\Sigma}_{-}}, \overline{\mathcal{C}}_{\hbar}^{-}\right)-2 \sum_{\beta \in K^{\eta}(\hbar)} \bar{\mu}_{\hbar}^{\bar{\Sigma}_{-}}\left(\overline{\mathcal{C}}_{\beta}^{-}\right) \log W_{\beta}^{-} .
$$

Thanks to Proposition 5.1, we know that

$$
p\left(\bar{\mu}_{\hbar}^{\bar{\Sigma}_{+}}, \overline{\mathcal{C}}_{\hbar}^{+}\right)+p\left(\bar{\mu}_{\hbar}^{\bar{\Sigma}_{-}}, \overline{\mathcal{C}}_{\hbar}^{-}\right) \geq-\log C-\left(1+\epsilon^{\prime}+4 \epsilon\right) n_{E}(\hbar),
$$

where $C$ is a constant that does not depend on $\hbar$.

Remark. This last inequality is a crucial step to prove Theorem 1.1. We will recall how one can get such a lower bound in Sect. 5. This inequality corresponds to Proposition 5.3 in [16]. The strategy of the proof is exactly the same except that we have to deal with quantum pressures and not quantum entropies (see Sect. 5). However, we can follow the same lines as in Sect. 5.3.2 in [16] and obtain a lower bound that depends on the bound from Theorem 4.1. At this point, there is a difference because Theorem 4.1 was proved in [4] for Anosov flows. In Sect. 4, we will show that the proof of this result from [4] can be adapted in the setting of nonpositively curved surfaces.

The partitions $\overline{\mathcal{C}}_{\hbar}^{+}$and $\overline{\mathcal{C}}_{\hbar}^{-}$are not exactly refined partitions of the suspension flow (as in definition (41) for instance). However, as in the Anosov setting, one can prove that they are refinements of "true refined partitions" of the suspension flow. A notable difference is that we will not consider time

\footnotetext{
${ }^{5}$ We refer the reader to Appendix B for the definition of $H$.
} 
1 of the suspension flow. Instead of it, we fix a large integer $N_{0}$ (such that ${ }^{6}$ $\left.\epsilon^{\prime} \ll 1 / N_{0} \ll \epsilon_{0}\right)$ and consider time $1 / N_{0}$ of the flow and its iterates. Precisely, as in [16], one can prove the following lemma:

Lemma 3.1. Let $N_{0}$ be a positive integer defined as previously. There exists an explicit partition $\overline{\mathcal{C}}_{N_{0}}^{+}$of $\bar{\Sigma}_{+}$, independent of $\hbar$ such that $\vee_{i=0}^{n_{E}(\hbar) N_{0}-1} \bar{\sigma}_{+}^{-\frac{i}{N_{0}}} \overline{\mathcal{C}}_{+}$ is a refinement of the partition $\overline{\mathcal{C}}_{\hbar}^{+}$. Moreover, let $n$ be a fixed positive integer. Then, an atom of the refined partition $\vee_{i=0}^{n-1} \bar{\sigma}_{+}^{-\frac{i}{N_{0}}} \overline{\mathcal{C}}_{+}$is of the form $[\alpha] \times B(\alpha)$, where $\alpha=\left(\alpha_{0}, \ldots, \alpha_{k}\right)$ is a $k+1$-uple such that $\left(\alpha_{0}, \ldots, \alpha_{k}\right)$ verifies $\frac{n}{N_{0}}(1-\epsilon) \leq$ $\sum_{j=0}^{k-1} f_{+}\left(\sigma_{+}^{j} \alpha\right) \leq \frac{n}{N_{0}}(1+\epsilon)$ and $B(\alpha)$ is a subinterval of $\left[0, f_{+}(\alpha)[\right.$.

Remark. This lemma is the exact analogue of Lemma 4.1 in [16] and its proof is the same: the only difference is that we consider times $1 / N_{0}$ instead of time 1. In particular, in the proof, the partition $\overline{\mathcal{C}}_{N_{0}}^{+}$is constructed from ${ }^{7} \tilde{I}^{\eta}\left(1 / N_{0}\right)$ and not from $\tilde{I}^{\eta}(1)$. We also underline that we have only stated the result in the case of $\sigma_{+}$. The same results holds for $\sigma_{-}$: there exists an adapted partition $\overline{\mathcal{C}}_{N_{0}}^{-}$with the same properties.

As in the Anosov case, we would like to use this lemma to rewrite the quantum pressure in terms of the pressure of a refined partition. To do this, we use basic properties of the classical entropy (see Appendix B) to find that

$$
H\left(\bar{\mu}_{\hbar}^{\bar{\Sigma}_{+}}, \overline{\mathcal{C}}_{\hbar}^{+}\right) \leq H_{N_{0} n_{E}(\hbar)}\left(\bar{\mu}_{\hbar}^{\bar{\Sigma}_{+}}, \bar{\sigma}_{+}^{\frac{1}{N_{0}}}, \overline{\mathcal{C}}_{N_{0}}^{+}\right)
$$

where $H_{n}($.$) is defined by (41). Consider now an atom A$ of the partition $\vee_{j=0}^{n_{E}(\hbar) N_{0}-1} \bar{\sigma}_{+}^{-\frac{j}{N_{0}}} \overline{\mathcal{C}}_{N_{0}}^{+}$. There exists an unique family $\left(\gamma_{0}, \ldots, \gamma_{n_{E}}(\hbar) N_{0}-1\right)$ in $\tilde{I}^{\eta}\left(1 / N_{0}\right)^{N_{0} n_{E}(\hbar)}$ corresponding to this atom and we define the associated weight as

$$
W_{A}^{+}:=\prod_{j=0}^{N_{0} n_{E}(\hbar)-1} W_{\gamma_{j}}^{+} .
$$

From Lemma 3.1, we know that for every such $A$, there exists $\alpha$ in $I^{\eta}(\hbar)$ such that $A$ is a subset of $[\alpha] \times\left[0, f_{+}(\alpha)[\right.$. From the proof of this lemma (see Sect. 5.2.3 in [16]), we know that $\alpha$ is of the form $\left(\tilde{\gamma}_{0}, \ldots, \tilde{\gamma}_{n_{E}(\hbar) N_{0}-1}\right)$ where every $\tilde{\gamma}_{j}$ is given by $\gamma_{j}$ where we have erased at most the last $b_{0} / \epsilon_{0}+1$ letters. In particular, this implies that

$$
W_{A}^{+} \leq \exp \left(2 N_{0} n_{E}(\hbar)\left(\frac{b_{0}}{\epsilon_{0}}+1\right) b_{0} \eta\right) W_{\alpha}^{+} .
$$

\footnotetext{
${ }^{6}$ To summarize the relations between the different parameters, we have $\frac{\epsilon}{4}<\epsilon^{\prime} \ll \frac{1}{N_{0}} \ll \epsilon_{0}$. Moreover $\eta$ depends on $\epsilon$ and $\epsilon_{0}$ and tends to 0 when $\epsilon$ tends to 0 and $\epsilon_{0}$ is fixed.

${ }^{7}$ We define $\tilde{I}^{\eta}(t)=\left\{\alpha=\left(\alpha_{0}, \ldots, \alpha_{k}\right): \sum_{i=1}^{k-2} f_{+}\left(\sigma_{+}^{i} \alpha\right) \leq t<\sum_{i=1}^{k-1} f_{+}\left(\sigma_{+}^{i} \alpha\right)\right\}$.
} 
Recall that we have taken $\left(\frac{b_{0}}{\epsilon_{0}}+1\right) b_{0} \eta \leq \epsilon / 2$. One can then write the following inequality

$$
\begin{aligned}
& -2 \sum_{\alpha \in I^{\eta}(\hbar)} \bar{\mu}_{\hbar}^{\bar{\Sigma}_{+}}\left(\overline{\mathcal{C}}_{\alpha}^{+}\right) \log W_{\alpha}^{+}
\end{aligned}
$$

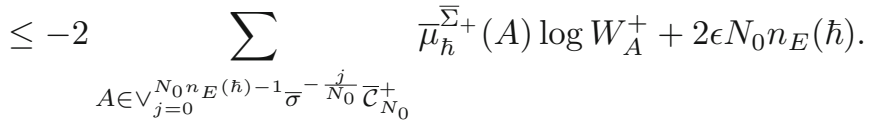

We introduce the refined pressure at time $n$

$$
\begin{aligned}
& p_{n}\left(\bar{\mu}_{\hbar}^{\bar{\Sigma}_{+}}, \bar{\sigma}_{+}^{\frac{1}{N_{0}}}, \overline{\mathcal{C}}_{N_{0}}^{+}\right) \\
& :=H_{n}\left(\bar{\mu}_{\hbar}^{\bar{\Sigma}_{+}}, \bar{\sigma}_{+}^{\frac{1}{N_{0}}}, \overline{\mathcal{C}}_{N_{0}}^{+}\right)-2 \sum_{A \in \vee_{j=0}^{n-1} \bar{\sigma}^{-} \frac{j}{N_{0}}} \overline{\mathcal{C}}_{N_{0}}^{+} \bar{\mu}_{\hbar}^{\bar{\Sigma}_{+}}(A) \log W_{A}^{+} .
\end{aligned}
$$

Finally, combining inequalities (24) and (25) with (23), we derive that

$$
\begin{aligned}
& -\log C-\left(1+\epsilon^{\prime}+4\left(1+N_{0}\right) \epsilon\right) n_{E}(\hbar) \\
& \quad \leq p_{n_{E}(\hbar) N_{0}}\left(\bar{\mu}_{\hbar}^{\bar{\Sigma}_{+}}, \bar{\sigma}_{+}^{\frac{1}{N_{0}}}, \overline{\mathcal{C}}_{N_{0}}^{+}\right)+p_{n_{E}(\hbar) N_{0}}\left(\bar{\mu}_{\hbar}^{\bar{\Sigma}_{-}}, \bar{\sigma}_{-}^{\frac{1}{N_{0}}}, \overline{\mathcal{C}}_{N_{0}}^{-}\right) .
\end{aligned}
$$

This estimate is crucial in our proof as we have derived from a quantum relation a lower bound on the classical pressure of a dynamical system associated to the geodesic flow.

\subsection{Subadditivity of the Quantum Pressure}

As in [16], we would like to let $\hbar$ tends to 0 in inequality (26). The main difficulty to do this is that everything depends on $\hbar$. In order to overcome this problem, we have to prove a subadditivity property for the quantum pressure:

Theorem 3.2. Let $\overline{\mathcal{C}}_{N_{0}}^{+}$be the partition of Lemma 3.1. There exists a function $R\left(n_{0}, \hbar\right)$ on $\mathbb{N} \times(0,1]$ and $R\left(N_{0}\right)$ independent of $n_{0}$ such that

$$
\forall n_{0} \in \mathbb{N}, \quad \limsup _{\hbar \rightarrow 0}\left|R\left(n_{0}, \hbar\right)\right|=R\left(N_{0}\right) .
$$

Moreover, for any $\hbar \in(0,1]$ and any $n_{0}, m \in \mathbb{N}$ such that $n_{0}+m \leq N_{0} n_{E}(\hbar)$, one has

$$
\begin{aligned}
p_{n_{0}+m}\left(\bar{\mu}_{\hbar}^{\bar{\Sigma}_{+}}, \bar{\sigma}^{\frac{1}{N_{0}}}, \overline{\mathcal{C}}_{N_{0}}^{+}\right) \leq & p_{n_{0}}\left(\bar{\mu}_{\hbar}^{\bar{\Sigma}_{+}}, \bar{\sigma}^{\frac{1}{N_{0}}}, \overline{\mathcal{C}}_{N_{0}}^{+}\right) \\
& +p_{m}\left(\bar{\mu}_{\hbar}^{\bar{\Sigma}_{+}}, \bar{\sigma}^{\frac{1}{N_{0}}}, \overline{\mathcal{C}}_{N_{0}}^{+}\right)+R\left(n_{0}, \hbar\right)
\end{aligned}
$$

Proof. To prove this subadditivity property, we will prove subadditivity of the quantum entropy and subadditivity of the pressure term. As in Sect. 6 from [16], we write for the entropy part that

$$
\begin{aligned}
& H_{n_{0}+m}\left(\bar{\mu}_{\hbar}^{\bar{\Sigma}_{+}}, \bar{\sigma}^{\frac{1}{N_{0}}}, \overline{\mathcal{C}}_{N_{0}}^{+}\right) \\
& \quad \leq H_{n_{0}}\left(\bar{\mu}_{\hbar}^{\bar{\Sigma}_{+}} \circ \bar{\sigma}_{+}^{-\frac{m}{N_{0}}}, \bar{\sigma}^{\frac{1}{N_{0}}}, \overline{\mathcal{C}}_{N_{0}}^{+}\right)+H_{m}\left(\bar{\mu}_{\hbar}^{\bar{\Sigma}_{+}}, \bar{\sigma}^{\frac{1}{N_{0}}}, \overline{\mathcal{C}}_{N_{0}}^{+}\right) .
\end{aligned}
$$


As in [16], we have to show that the measure of the atoms of the partition is almost invariant under $\bar{\sigma}_{+}^{\frac{1}{N_{0}}}$ for the range of times we have considered (Proposition 6.1 in [16]). Consider now the pressure term in the quantum pressure. Using the multiplicative structure of the $W_{A}^{+}$, one has

$$
\begin{aligned}
& \sum_{A \in \vee_{j=0}^{n_{0}+m-1} \bar{\sigma}^{-\frac{j}{N_{0}}} \overline{\mathcal{C}}_{N_{0}}^{+}} \bar{\mu}_{\hbar}^{\bar{\Sigma}_{+}}(A) \log W_{A}^{+} \\
& =\sum_{A \in \vee_{j=0}^{m-1} \bar{\sigma}^{-\frac{j}{N_{0}}} \overline{\mathcal{C}}_{N_{0}}^{+}} \bar{\mu}_{\hbar}^{\bar{\Sigma}_{+}}(A) \log W_{A}^{+} \\
& +\sum_{A \in \mathrm{V}_{j=0}^{n_{0}-1} \bar{\sigma}^{-\frac{j}{N_{0}}} \overline{\mathcal{C}}_{N_{0}}^{+}} \bar{\mu}_{\hbar}^{\bar{\Sigma}_{+}}\left(\bar{\sigma}_{+}^{-\frac{m}{N_{0}}} A\right) \log W_{A}^{+} .
\end{aligned}
$$

So, once more, the additivity property of the pressure term derives from the almost invariance of the measure for the range of times we consider. ${ }^{8}$ Precisely, according to the last two inequalities, we only need to verify that Proposition 6.1 in [16] remains true for the partition $\overline{\mathcal{C}}_{N_{0}}^{+}$in the setting of surfaces of nonpositive curvature. We will not reproduce the proof here which is similar except that we consider time $1 / N_{0}$ instead of 1 and that we look at surfaces of nonpositive curvature. The first difference is not a problem and the proof from [16] can be adapted straightforward. The main difference comes from the fact that the geometric situation is slightly different. We will briefly explain here which points need to be modified in this new setting.

We recall that Proposition 6.1 in [16] relied on a theorem for products of pseudodifferential operators (Theorem 7.1 in [16]) and we need to verify that the proof we gave still works in the case of surfaces of nonpositive curvature. The key point of the proof of this theorem is that in the allowed range of times, $\left\|d_{\rho} g^{t}\right\|$ is bounded by some $\hbar^{-\nu}$ (with $\nu<1 / 2$ ) (see Sect. 7.2 in [16]). Precisely, following Sects. 6 and 7 in [16], we need to verify that this bound on the growth of $\left\|d_{\rho} g^{t}\right\|$ holds for $\rho$ in $T^{*} \Omega_{\alpha_{0}} \cap \cdots g^{(k-1) \eta} T^{*} \Omega_{\alpha_{k-1}} \cap \mathcal{E}^{\theta}$ (where $\alpha$ satisfies $\sum_{j=0}^{k-1} f_{+}\left(\sigma_{+}^{j} \alpha\right) \leq \frac{n_{E}(\hbar)}{2}$ ) and for $0 \leq t \leq k \eta$. In fact, if we take $\mathrm{Op}_{\hbar}(\chi)$ to be a "good" truncation operator in a neighborhood of $S^{*} M$, it allows to verify that $P_{\alpha_{k-1}}((k-1) \eta) \cdots P_{\alpha_{0}} \mathrm{Op}_{\hbar}(\chi)$ satisfies the usual rules of pseudodifferential calculus (see Theorem 7.1 in [16]) and then to derive the property of almost invariance of the measure $\bar{\mu}_{\hbar} \bar{\Sigma}_{+}$(Proposition 6.1 in [16]).

From Sect. 2.1.3 and if we take $\delta^{\prime}=\epsilon_{0} \epsilon$, we find that $\left\|d_{\rho} g^{k \eta}\right\|$ is bounded by $C e^{k \eta \epsilon_{0} \epsilon} e^{\int_{0}^{k \eta} U_{\rho}^{u}(s) \mathrm{d} s}$ (with $C$ uniform in $\rho$ ). For the allowed words, $2 e^{k \eta \epsilon_{0} \epsilon}$ is of order $\hbar^{-\epsilon}\left(\right.$ as $\left.k \eta \epsilon_{0} \leq 1 / 2 n_{E}(\hbar)\right)$. To conclude, we can estimate:

$$
\left|\int_{0}^{k \eta} U_{\rho}^{u}(s) \mathrm{d} s-\sum_{j=0}^{k-1} f\left(\sigma^{j} \alpha\right)\right| \leq \sum_{j=0}^{k-1}\left|\int_{j \eta}^{(j+1) \eta} U_{\rho}^{u}(s) \mathrm{d} s-f\left(\sigma^{j} \alpha\right)\right| .
$$

\footnotetext{
${ }^{8}$ We underline that $R\left(N_{0}\right)$ will be equal to $\sup _{A \in \overline{\mathcal{C}}_{N_{0}}^{+}} \log W_{A}^{+}$which only depends on $N_{0}$.
} 
To bound this sum, we can use the continuity of $U^{u}$ (see inequality (6)) to show that this quantity is bounded by $\epsilon|\log \hbar|$. By definition of the allowed words $\alpha$, we know that $\sum_{j=0}^{k-1} f\left(\sigma^{j} \alpha\right) \leq 1 / 2 n_{E}(\hbar)$. This allows to conclude that $\left|d_{\rho} g^{t}\right|$ is bounded by some $C \hbar^{-\nu}$ (with $C$ independent of $\rho$ and $\nu<1 / 2$ ).

Remark. We underline that here we need to use the specific properties of surfaces of nonpositive curvature to prove this theorem. It is not really surprising that Theorem 7.1 from [16] can be extended in our setting as the situation can only be less "chaotic". We also mention that we have to use the continuity of $U^{u}(\rho)$ which is for instance false for surfaces without conjugate points [5].

\subsection{The Conclusion}

3.4.1. The Semiclassical Parameter Tends to 0. Thanks to the subadditivity property of the quantum pressure, we can proceed as in [16] and write, for a fixed $n_{0}$, the euclidean division $N_{0} n_{E}(\hbar)=q n_{0}+r$. We find, after applying the subadditivity property and letting $\hbar$ tends to 0 ,

$$
\begin{aligned}
& -\frac{R\left(N_{0}\right)}{n_{0}}-\frac{1}{N_{0}}\left(1+\epsilon^{\prime}+4\left(1+N_{0}\right) \epsilon\right) \\
& \quad \leq \frac{1}{n_{0}}\left(p_{n_{0}}\left(\bar{\mu}^{\bar{\Sigma}_{+}}, \bar{\sigma}_{+}^{\frac{1}{N_{0}}}, \overline{\mathcal{C}}_{N_{0}}^{+}\right)+p_{n_{0}}\left(\bar{\mu}^{\bar{\Sigma}_{-}}, \bar{\sigma}_{-}^{\frac{1}{N_{0}}}, \overline{\mathcal{C}}_{N_{0}}^{-}\right)\right) .
\end{aligned}
$$

As in $[2,4,16]$, we can replace the smooth partitions by true partitions of the manifold in the previous inequality. We would like now to transform the previous inequality on the metric pressure into an inequality on the Kolmogorov-Sinai entropy. To do this, we write the multiplicative property of $W_{A}^{+}$and we use the fact that $\overline{\mathcal{C}}_{N_{0}}^{+}$is a partition of $\bar{\Sigma}_{+}$. It allows us to derive that

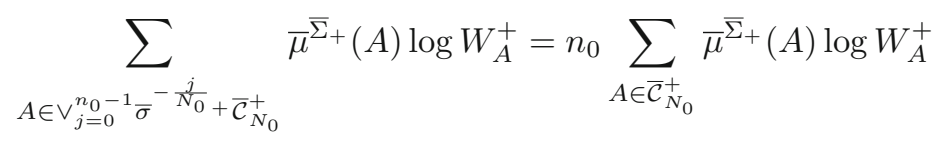

The same property holds for the backward side. After letting $n_{0}$ tends to infinity, we find that

$$
\begin{aligned}
& -\frac{1}{N_{0}}\left(1+\epsilon^{\prime}+4\left(1+N_{0}\right) \epsilon\right) \\
& \quad+2\left(\sum_{A \in \overline{\mathcal{C}}_{N_{0}}^{+}} \bar{\mu}^{\bar{\Sigma}_{+}}(A) \log W_{A}^{+}+\sum_{A \in \overline{\mathcal{C}}_{N_{0}}^{-}} \bar{\mu}^{\bar{\Sigma}_{-}}(A) \log W_{A}^{-}\right) \\
& \quad \leq \frac{1}{N_{0}}\left(h_{K S}\left(\bar{\mu}^{\bar{\Sigma}_{+}}, \bar{\sigma}_{+}\right)+h_{K S}\left(\bar{\mu}^{\bar{\Sigma}_{-}}, \bar{\sigma}_{-}\right)\right) .
\end{aligned}
$$

3.4.2. Lower Bound on $\sum_{A \in \overline{\mathcal{C}}_{N_{0}}^{+}} \overline{\boldsymbol{\mu}}^{\overline{\boldsymbol{\Sigma}}^{+}}(\boldsymbol{A}) \log \boldsymbol{W}_{\boldsymbol{A}}^{+}$. Before applying Abramov theorem in inequality (27), we would like to give a lower bound on the pressure term in this inequality. Precisely, we know that, by construction of $\overline{\mathcal{C}}_{N_{0}}^{+}$and 
by invariance of the measure $\mu^{\Sigma_{+}}$, one has

$$
\sum_{A \in \overline{\mathcal{C}}_{N_{0}}^{+}} \bar{\mu}^{\bar{\Sigma}_{+}}(A) \log W_{A}^{+}=\frac{1}{\int_{\Sigma_{+}} f_{0} \mathrm{~d} \mu^{\Sigma_{+}}} \sum_{\gamma \in \tilde{I}^{\eta}\left(1 / N_{0}\right)} f_{0}(\gamma) \mu^{\Sigma_{+}}([\gamma]) \log W_{\gamma}^{+} .
$$

To obtain a lower bound on this quantity, we use the notations of Sect. 2.1 and introduce, for $\rho \in S^{*} M$, the application

$$
F_{0}(\rho):=\sum_{\gamma \in \tilde{I}^{\eta}\left(1 / N_{0}\right)} f_{0}(\gamma) \log W_{\gamma}^{+} \mathbf{1}_{O_{\gamma_{0}}}(\rho) \cdots \mathbf{1}_{O_{\gamma_{k}}} \circ g^{k \eta}(\rho) .
$$

This allows us to rewrite

$$
\sum_{\gamma \in \tilde{I}^{\eta}\left(1 / N_{0}\right)} f_{0}(\gamma) \mu^{\Sigma}([\gamma]) \log W_{\gamma}^{+}=\int_{S^{*} M} F_{0}(\rho) \mathrm{d} \mu(\rho)
$$

Define also

$$
X_{0}^{(\eta)}:=\left\{\rho \in S^{*} M: \forall 0 \leq t \leq \frac{1}{N_{0} \epsilon_{0}}+\eta, U^{u}\left(g^{t} \rho\right)>2 \epsilon_{0}\right\} .
$$

We can verify that

$$
F_{0}(\rho) \geq \frac{1}{2 N_{0}} \sum_{\gamma_{0}, \gamma_{1}} f_{0}(\gamma) \mathbf{1}_{X_{0}^{(\eta)}}(\rho) \mathbf{1}_{O_{\gamma_{0}}}(\rho) \mathbf{1}_{O_{\gamma_{1}}} \circ g^{\eta}(\rho)
$$

for all $\rho$ in $\mathcal{E}^{\theta}$. In order to prove this property, we can restrict ourselves to the case: $\rho \in X_{0}^{(\eta)}$ (otherwise the inequality is trivial). In this case, $F_{0}(\rho)=$ $f_{+}(\gamma) \log W_{\gamma}^{+}$, where $\gamma$ is the unique element in $\tilde{I}^{\eta}\left(1 / N_{0}\right)$ such that $\rho$ belongs to $O_{\gamma_{0}} \cap \cdots g^{-k \eta} O_{\gamma_{k}}$. As $\gamma$ belongs to $\tilde{I}^{\eta}\left(1 / N_{0}\right)$, it satisfies

$$
\sum_{j=1}^{k-2} f_{+}\left(\sigma^{j} \gamma\right) \leq \frac{1}{N_{0}}<\sum_{j=1}^{k-1} f_{+}\left(\sigma^{j} \gamma\right)
$$

As $f_{+} \geq \eta \epsilon_{0}$, one has $(k-2) \eta \leq 1 /\left(N_{0} \epsilon_{0}\right)$. Using the fact that $\rho$ belongs to $X_{0}^{(\eta)} \cap O_{\gamma_{0}} \cap \cdots g^{-k \eta} O_{\gamma_{k}}$ and using the relation of continuity (4), we find that for every $1 \leq j \leq k-1, f_{+}\left(\sigma^{j} \gamma\right)=f\left(\sigma^{j} \gamma\right)$. In particular, one has

$$
\log W_{\gamma}^{+}=\frac{1}{2} \sum_{j=1}^{k-1} f_{+}\left(\sigma^{j} \gamma\right) \geq \frac{1}{2 N_{0}} .
$$

Then, we can derive

$$
\sum_{A \in \overline{\mathcal{C}}_{N_{0}}^{+}} \bar{\mu}^{\bar{\Sigma}_{+}}(A) \log W_{A}^{+} \geq \frac{\sum_{\gamma_{0}, \gamma_{1}} f_{0}(\gamma) \mu\left(X_{0}^{(\eta)} \cap O_{\gamma_{0}} \cap g^{-\eta} O_{\gamma_{1}}\right)}{2 N_{0} \int_{\Sigma_{+}} f_{+} \mathrm{d} \mu^{\Sigma_{+}}} .
$$

We underline that the same lower bound holds for $\sum_{A \in \overline{\mathcal{C}}_{N_{0}}^{-}} \bar{\mu}^{\bar{\Sigma}_{-}}(A) \log W_{A}^{-}$. 
3.4.3. Applying Abramov Theorem. We use this last property in inequality (27) and combine it with the Abramov theorem [1] (see relation (44)). We find that the Kolmogorov Sinai entropy $\eta h_{K S}(\mu, g)$ is bounded from below by

$$
\sum_{\gamma=\left(\gamma_{0}, \gamma_{1}\right)} f_{0}(\gamma)\left(\Gamma\left(\epsilon, \epsilon^{\prime}, N_{0}\right) \mu^{\Sigma}([\gamma])+\mu\left(X_{0}^{(\eta)} \cap O_{\gamma_{0}} \cap g^{-\eta} O_{\gamma_{1}}\right)\right),
$$

where

$$
\Gamma\left(\epsilon, \epsilon^{\prime}, N_{0}\right):=-\frac{1}{2}+\left(-\frac{\epsilon^{\prime}}{2}-2\left(1+N_{0}\right) \epsilon\right) .
$$

3.4.4. The Different Small Parameters Tend to 0. We have obtained a lower bound on the Kolmogorov-Sinai entropy of the measure $\mu$. This lower bound depends on several small parameters that are linked to each other in the following way:

$$
\epsilon<4 \epsilon^{\prime} \ll \frac{1}{N_{0}} \ll \epsilon_{0} .
$$

Moreover the small parameter $\eta$ depends on $\epsilon$ and $\epsilon_{0}$. For a fixed $\epsilon_{0}$, it tends to 0 when $\epsilon$ tends to 0 . We have now to be careful to transform our lower bound on the entropy of $\mu$ into the expected lower bound. First, we let the diameter of the partition tends to 0 (and then $\theta$ to 0 ) and we divide by $\eta$. This gives us

$$
\int_{S^{*} M}\left(\Gamma\left(\epsilon, \epsilon^{\prime}, N_{0}\right)+\mathbf{1}_{X_{0}^{(\eta)}}(\rho)\right) U_{0}^{u}(\rho) \mathrm{d} \mu(\rho) \leq h_{K S}(\mu, g) .
$$

Finally, we let $\epsilon$ and $\epsilon^{\prime}$ tend to 0 (in this order). We obtain the following bound on the entropy of $\mu$ :

$$
-\frac{1}{2} \int_{S^{*} M} U_{0}^{u}(\rho) \mathrm{d} \mu(\rho)+\int_{S^{*} M} U_{0}^{u}(\rho) \mathbf{1}_{X_{0}^{(0)}}(\rho) \mathrm{d} \mu(\rho) \leq h_{K S}(\mu, g) .
$$

We let now $N_{0}$ tend to infinity and then $\epsilon_{0}$ tend to 0 (in this order). We find the expected lower bound, i.e.

$$
\frac{1}{2} \int_{S^{*} M} U^{u}(\rho) \mathrm{d} \mu(\rho) \leq h_{K S}(\mu, g) .
$$

\section{Proof of the Main Estimate from [4]}

In the previous section, we have been able to apply the method we used for Anosov surfaces in order to prove Theorem 1.1. As in [16], the strategy relied on a careful adaptation of an uncertainty principle. In particular, to derive inequality (23) (Sect. 5), we have to use the following equivalent of Theorem 3.1 from [3]: 
Theorem 4.1. Let $M$ be a surface of nonpositive sectional curvature and $\epsilon, \epsilon_{0}$ and $\eta$ be small positive parameters as in Sect. 2.2. For every $\mathcal{K}>0\left(\mathcal{K} \leq C_{\delta_{0}}\right)$, there exists $\hbar_{\mathcal{K}}$ and $C_{\mathcal{K}}\left(\epsilon, \eta, \epsilon_{0}\right)$ such that uniformly for all $\hbar \leq \hbar_{\mathcal{K}}$, for all $k \leq \mathcal{K}|\log \hbar|$, for all $\alpha=\left(\alpha_{0}, \ldots, \alpha_{k}\right)$,

$$
\begin{aligned}
& \left\|P_{\alpha_{k}} U^{\eta} P_{\alpha_{k-1}} \cdots U^{\eta} P_{\alpha_{0}} O p_{\hbar}\left(\chi^{(k)}\right)\right\|_{L^{2}(M)} \\
& \quad \leq C_{\mathcal{K}}\left(\epsilon, \eta, \epsilon_{0}\right) \hbar^{-\frac{1}{2}-c \delta_{0}} e^{2 k \eta \epsilon_{0} \epsilon} \exp \left(-\frac{1}{2} \sum_{j=0}^{k-1} f\left(\sigma_{+}^{j} \alpha\right)\right),
\end{aligned}
$$

where $c$ depends only on the riemannian manifold $M$.

Remark. We underline two facts about this theorem. The first one is that $\mathrm{Op}_{\hbar}\left(\chi^{(k)}\right)$ is a cutoff operator that was already defined in [16, Paragraph 5.3] and in the appendix of [4] (we describe briefly its construction in Sect. 5.1). The second one is that it is function $f$ and not $f_{+}$that appears in the upper bound.

This theorem is the analogue for surfaces of nonpositive curvature of a theorem from [4]. As the geometric situation is slightly different from [4], we will recall the main lines of the proof where the geometric properties appear and focus on the differences. We refer the reader to [4] for the details. In [4], the proof of the analogue of Theorem 4.1 (Sect. 3 and more precisely Corollary 3.5) relies on a study of the action of $P_{\alpha_{k}} U^{\eta} P_{\alpha_{k-1}} \cdots U^{\eta} P_{\alpha_{0}}$ on a particular family of Lagrangian states. This reduction was possible because of the introduction of the cutoffs operators $\mathrm{Op}_{\hbar}\left(\chi^{(k)}\right)$ (see Sect. 3 in [4] for the details).

\subsection{Evolution of a WKB State}

Consider $u_{\hbar}(0, x)=a_{\hbar}(0, x) e^{\frac{\imath}{\hbar} S(0, x)}$ a Lagrangian state, where $a_{\hbar}(0, \bullet)$ and $S(0, \bullet)$ are smooth functions on a subset $\Omega$ in $M$ and $a_{\hbar}(0, \bullet) \sim \sum_{k} \hbar^{k} a_{k}(0, \bullet)$. This represents a Lagrangian state which is supported on the Lagrangian manifold $\mathcal{L}(0):=\left\{\left(x, d_{x} S(0, x): x \in \Omega\right\}\right.$. According to [4], if we are able to understand the action of $P_{\alpha_{k}} U^{\eta} P_{\alpha_{k-1}} \cdots U^{\eta} P_{\alpha_{0}}$ on Lagrangian states (with specific initial Lagrangian manifolds: see next paragraph), then we can derive our main theorem. A strategy to estimate this action is to use a WKB Ansatz. Recall that if we note $\tilde{u}(t):=U^{t} u_{\hbar}(0)$, then, for any integer $N$, the state $\tilde{u}(t)$ can be approximated to order $N$ by a Lagrangian state $u(t)$ of the form

$$
u(t, x):=e^{\frac{2}{\hbar} S(t, x)} a_{\hbar}(t, x)=e^{\frac{2}{\hbar} S(t, x)} \sum_{K=0}^{N-1} \hbar^{k} a_{k}(t, x) .
$$

As $u$ is supposed to solve $\imath \hbar \frac{\Delta}{2} u=\partial_{t} u$ (up to an error term of order $N$ ), we know that $S(t, x)$ and the $a_{k}(t, x)$ satisfy several partial differential equations. In particular, $S(t, x)$ must solve the Hamilton-Jacobi equation

$$
\frac{\partial S}{\partial t}+H\left(x, d_{x} S\right)=0
$$


Assume that, on a certain time interval (for instance $s \in[0, \eta]$ ), the above equations have a well defined smooth solution $S(s, x)$, meaning that the transported Lagrangian manifold $\mathcal{L}(s)=g^{s} \mathcal{L}(0)$ is of the form $\left\{\left(x, d_{x} S(s, x)\right)\right\}$, where $S(s)$ is a smooth function on the open set $\pi \mathcal{L}(s)$.

As in [4], we shall say that a Lagrangian manifold $\mathcal{L}$ is "projectible" if the projection $\pi: \mathcal{L} \rightarrow M$ is a diffeomorphism onto its image. If the projection of $\mathcal{L}$ on $M$ is simply connected, $\mathcal{L}$ is the graph of $d S$ for some function $S$ : we say that $\mathcal{L}$ is generated by $S$.

Suppose now that, for $s \in[0, \eta]$, the Lagrangian $\mathcal{L}(s)$ is "projectible". Then, this family of Lagrangian manifolds defines an induced flow on $M$, i.e.

$$
g_{S(s)}^{t}: x \in \pi \mathcal{L}(s) \mapsto \pi g^{t}\left(x, d_{x} S(s, x)\right) \in \pi \mathcal{L}(s+t) .
$$

This flow satisfies a property of semi-group: $g_{S(s+\tau)}^{t} \circ g_{S(s)}^{\tau}=g_{S(s)}^{t+\tau}$. Using this flow, we define an operator that sends functions on $\pi \mathcal{L}(s)$ into functions on $\pi \mathcal{L}(s+t)$ :

$$
T_{S(s)}^{t}(a)(x):=a \circ g_{S(s+t)}^{-t}(x)\left(J_{S(s+t)}^{-t}(x)\right)^{\frac{1}{2}},
$$

where $J_{S(s)}^{t}(x)$ is the Jacobian of the map $g_{S(s)}^{t}$ at point $x$ (w.r.t. the riemannian volume). This operator allows to give an explicit expression for all the $a_{k}(t)$ [4], i.e.

$$
a_{k}(t):=T_{S(0)}^{t} a_{0}(0) \quad \text { and } \quad a_{k}(t):=T_{S(0)}^{t} a_{k}(0)+\int_{0}^{t} T_{S(s)}^{t-s}\left(\frac{\imath \Delta a_{k-1}(s)}{2}\right) \mathrm{d} s .
$$

Regarding the details of the proof in [4], we know that there are two main points where the geometric properties of the manifold are used:

- the evolution of the Lagrangian manifold under the action of the operator $P_{\alpha_{k}} U^{\eta} P_{\alpha_{k-1}} \cdots U^{\eta} P_{\alpha_{0}}$ (Sect. 3.4.1 in [4]);

- the value of $J_{S(0)}^{t}$ for large $t$ (Sect. 3.4.2 in [4]).

We will discuss these two points in the two following paragraphs. We will recall what was proved for these two questions in Sect. 3.4 of [4] and see how it can be translated in the setting of surfaces of nonpositive curvature.

\subsection{Evolution of the Lagrangian Manifolds}

The first thing we need to understand is how the Lagrangian manifolds evolve under the action of the operator $P_{\alpha_{k}} U^{\eta} P_{\alpha_{k-1}} \cdots U^{\eta} P_{\alpha_{0}}$. According to [4], we know that the introduction of the cutoff operator $\mathrm{Op}_{\hbar}(\chi)$ implies that we can restrict ourselves to a particular family of Lagrangian states. Precisely, we fix some small parameter $\eta_{1}$ and we know that they must be localized on a piece of Lagrangian manifold $\mathcal{L}^{0}(0)$ which is included in the set $\cup_{|\tau| \leq \eta} g^{\tau} S_{z, \eta_{1}}^{*} M$ (where $\left.S_{z, \eta_{1}}^{*} M:=\left\{(z, \xi):\|\xi\|_{z}^{2}=1+2 \eta_{1}\right\}\right)$. If we follow the method developed in [4], we are given a sequence of Lagrangian manifolds $\mathcal{L}^{j}(0)$ as follows:

$$
\forall t \in[0, \eta], \forall j, \mathcal{L}^{0}(t):=g^{t} \mathcal{L}^{0}(0) \quad \text { and } \quad \mathcal{L}^{j}(t):=g^{t}\left(\mathcal{L}^{j-1}(\eta) \cap T^{*} \Omega_{\alpha_{j}}\right) .
$$


The manifold $\mathcal{L}^{j}(0)$ is obtained after performing $P_{\alpha_{j}} U^{\eta} P_{\alpha_{k-1}} \cdots U^{\eta} P_{\alpha_{0}}$ on the initial Lagrangian state. To show that the procedure from [4] is consistent (i.e. performing several WKB Ansatz), we need to verify that the Lagrangian manifold $\mathcal{L}^{j}(t)$ does not develop caustics and remains "projectible". The only geometric properties which were used to derive these two properties were:

- $M$ has no conjugate points (to derive that $S^{j}$ will not develop caustics);

- the injectivity radius is larger than 2 (to ensure the "projectible" property).

In our setting, these two properties remain true (in particular, a surface of nonpositive curvature has no conjugate points $[11,19])$. Finally, we underline that, thanks to the construction of the strong unstable foliation for surfaces of nonpositive curvature, any vector in $S_{z, \eta_{1}}^{*} M$ becomes uniformly close to the unstable subspace under the action of $d_{\rho} g^{t}$ (see Lemma 2.1). As a consequence, under the geodesic flow, a piece of sphere becomes uniformly close to the unstable foliation as $j$ tends to infinity. This point is the main difference with [4]. In fact, if we consider an Anosov geodesic flow, we have the stronger property that a piece of sphere becomes exponentially close to the unstable foliation, as $j$ tends to infinity. However, we will check that this property is sufficient for our needs.

Remark. At this point of the proof, we can ask about an extension of these results to manifolds without conjugate points. According to [19], the "uniform divergence" property (given by Lemma 2.1) is true for surfaces without conjugate points and so a piece of sphere also becomes uniformly close to the unstable foliation in this more general setting. So this crucial aspect of the proof can be transposed in the setting of surfaces without conjugate points. In the next paragraph, we will use an additional argument which is specific to surfaces of nonpositive curvature.

\subsection{Estimates on the Induced Jacobian}

As was already mentioned, the Jacobian $J_{S^{j}}^{t}$ of the map $g_{s_{j}}^{t}$ appears in the WKB expansion of a Lagrangian state evolved under the action of the operator $P_{\alpha_{j}} U^{\eta} P_{\alpha_{j-1}} \cdots U^{\eta} P_{\alpha_{0}}$. Precisely, by iterating the WKB Ansatz, we have to estimate the following quantity (see Eq. (3.22) in [4]):

$$
J_{k}(x):=\left(J_{S^{k-1}}^{-\eta}(x) J_{S^{k-2}}^{-\eta}\left(g_{S^{k}}^{-\eta}(x)\right) \cdots J_{S^{1}}^{-\eta}\left(g_{S^{k}}^{(-k+2) \eta}(x)\right)\right)^{\frac{1}{2}} .
$$

This Jacobian appears in each term of the WKB expansion of a Lagrangian state evolved under the operator $P_{\alpha_{k}} U^{\eta} P_{\alpha_{k-1}} \cdots U^{\eta} P_{\alpha_{0}}$ as every $a_{p}$ in the expansion is defined using the operator $T_{S(.)}^{t}$ (see Definitions (30) and (31)). It is necessary to provide a way to bound this quantity as it will appear in the control of every derivatives of the WKB expansion. According to the proof in [4], if we are able to bound uniformly this quantity, the bound we will obtain is the one that will appear in Theorem 4.1. This point of the proof is the main difference with the proof in the Anosov case. Our goal in this paragraph is to provide an upper bound on (32). The quantity $J_{k}(x)$ can be rewritten as 


$$
\exp \left(\frac{1}{2}\left(\log J_{S^{k-1}}^{-\eta}(x)+\log J_{S^{k-2}}^{-\eta}\left(g_{S^{k}}^{-\eta}(x)\right) \cdots+\log J_{S^{1}}^{-\eta}\left(g_{S^{k}}^{(-k+2) \eta}(x)\right)\right)\right) .
$$

As the Lagrangian $\mathcal{L}^{j}$ become uniformly close to the unstable foliation when $j$ tends to infinity, we know that, for every $\varepsilon^{\prime}>0$, there exists some integer $j\left(\eta, \varepsilon^{\prime}\right)$ such that

$$
\forall j \geq j\left(\eta, \varepsilon^{\prime}\right), \quad \forall \rho=(x, \xi) \in \mathcal{L}^{j}(0),\left|\log J_{S^{j}}^{-\eta}(x)-\log J_{S^{u}(\rho)}^{-\eta}(x)\right| \leq \varepsilon^{\prime},
$$

where $S^{u}(\rho)$ generates the local unstable manifold at point $\rho$ (which is a Lagrangian submanifold). Therefore, we find that there exists a constant $C\left(\varepsilon^{\prime}, \eta\right)$ (depending only on $\varepsilon^{\prime}$ and $\eta$ ) such that, uniformly with respect to $k$ and to $\rho$ in $\mathcal{L}^{k}(0)$,

$$
J_{k}(x) \leq C\left(\varepsilon^{\prime}, \eta\right) e^{k \varepsilon^{\prime}} \prod_{j=0}^{k-1} J_{S^{u}\left(g^{(-j+1) \eta} \rho\right)}^{-\eta}\left(g_{S^{k}}^{(-j+1) \eta}(x)\right)=C\left(\varepsilon^{\prime}, \eta\right) e^{k \varepsilon^{\prime}} J_{S^{u}(\rho)}^{(1-k) \eta}(x) .
$$

In the following, we will take $\varepsilon^{\prime}=\eta \epsilon \epsilon_{0}$. The Jacobian $J_{S^{u}(\rho)}^{-\eta}$ measures the contraction of $g^{-\eta}$ along the unstable direction. From the construction of the unstable Riccati solution $U_{\rho}^{u}(s)$, we know that $U_{\rho}^{u}(s)$ also measures the contraction of $g^{-\eta}$ along $E^{u}(\rho)$. In fact, according to Sect. 2.1, one has

$$
\left\|d_{\rho} g_{\mid E^{u}(\rho)}^{-t}\right\| \leq \sqrt{1+K_{0}} e^{\int_{0}^{-t} U_{\rho}^{u}(s) \mathrm{d} s} .
$$

As a consequence, there exists an uniform constant $C$ (depending only on the manifold) such that:

$$
J_{S^{u}(\rho)}^{(1-k) \eta}(x) \leq C e^{\int_{0}^{(1-k) \eta}} U_{\rho}^{u}(s) \mathrm{d} s .
$$

Using then relation (6) between the discrete Riccati solution $f$ and the continuous one, we find that there exists a constant $C\left(\epsilon, \eta, \epsilon_{0}\right)$ such that, uniformly in $k$,

$$
\sup _{x \in \pi \mathcal{L}^{k}(0)} J_{k}(x) \leq C\left(\epsilon, \eta, \epsilon_{0}\right) e^{2 k \eta \epsilon \epsilon_{0}} \exp \left(-\frac{1}{2} \sum_{j=0}^{k-1} f\left(\sigma^{j} \alpha\right)\right) .
$$

Finally, this last inequality gives us a bound on the quantity (32). This estimate is not as sharp as the one derived in [4] (Eq. 3.23 for instance) however it is sufficient as the correction term is not too large: it is of order $\hbar^{-\epsilon}$.

Remark. We underline that we used the continuity of $U^{u}$ to go from the continuous representation of the upper bound of $J_{k}$ to the one in terms of the discrete Riccati solution. This property fails for surfaces without conjugate points [5].

\section{Applying the Uncertainty Principle for Quantum Pressures}

In this section, we would like to prove inequality (23) which was a crucial step of our proof. To do this, we follow the same lines as in [16, Sect. 5.3] and prove the following proposition: 
Proposition 5.1. With the notations of Sect. 3, one has:

$$
p\left(\bar{\mu}_{\hbar}^{\bar{\Sigma}_{+}}, \overline{\mathcal{C}}_{\hbar}^{+}\right)+p\left(\bar{\mu}_{\hbar}^{\bar{\Sigma}_{-}}, \overline{\mathcal{C}}_{\hbar}^{-}\right) \geq-\log C-\left(1+\epsilon^{\prime}+4 \epsilon\right) n_{E}(\hbar)
$$

where $p$ is defined by (21) and where $C \in \mathbb{R}_{+}^{*}$ does not depend on $\hbar$ (but depends on the other parameters $\left.\left(\epsilon, \epsilon_{0}, \eta\right)\right)$.

To prove this result, we will proceed in three steps. First, we will introduce an energy cutoff in order to get the sharpest bound as possible in our application of the uncertainty principle. Then, we will apply the uncertainty principle and derive a lower bound on $p\left(\bar{\mu}_{\hbar}^{\bar{\Sigma}_{+}}, \overline{\mathcal{C}}_{\hbar}^{+}\right)+p\left(\bar{\mu}_{\hbar}^{\bar{\Sigma}_{-}}, \overline{\mathcal{C}}_{\hbar}^{-}\right)$. Finally, we will use the estimate of Theorem 4.1 to conclude.

\subsection{Energy Cutoff}

Before applying the uncertainty principle, we proceed to sharp energy cutoffs so as to get precise lower bounds on the quantum pressure (as it was done in [2-4]). These cutoffs are made in our microlocal analysis in order to get as good exponential decrease as possible of the norm of the refined quantum partition. This cutoff in energy is possible because even if the distributions $\mu_{\hbar}$ are defined on $T^{*} M$, they concentrate on the energy layer $S^{*} M$. The following energy localization is made in a way to compactify the phase space and in order to preserve the semiclassical measure.

Let $\delta_{0}$ be a positive number less than 1 and $\chi_{\delta_{0}}(t)$ in $\mathcal{C}^{\infty}(\mathbb{R},[0,1])$. Moreover, $\chi_{\delta_{0}}(t)=1$ for $|t| \leq e^{-\delta_{0} / 2}$ and $\chi_{\delta_{0}}(t)=0$ for $|t| \geq 1$. As in [4], the sharp $\hbar$-dependent cutoffs are then defined in the following way:

$\forall \hbar \in(0,1), \forall n \in \mathbb{N}, \forall \rho \in T^{*} M, \chi^{(n)}(\rho, \hbar):=\chi_{\delta_{0}}\left(e^{-n \delta_{0}} \hbar^{-1+\delta_{0}}(H(\rho)-1 / 2)\right)$.

For $n$ fixed, the cutoff $\chi^{(n)}$ is localized in an energy interval of size $2 e^{n \delta_{0}} \hbar^{1-\delta_{0}}$ centered around the energy layer $\mathcal{E}$. In this paper, indices $n$ will satisfy $2 e^{n \delta_{0}} \hbar^{1-\delta_{0}} \ll 1$. It implies that the widest cutoff is supported in an energy interval of microscopic length and that $n \leq K_{\delta_{0}}|\log \hbar|$, where $K_{\delta_{0}} \leq \delta_{0}^{-1}$. Using then a non standard pseudodifferential calculus (see [4] for a brief reminder of the procedure from [21]), one can quantize these cutoffs into pseudodifferential operators. We will denote $\operatorname{Op}\left(\chi^{(n)}\right)$ the quantization of $\chi^{(n)}$. The main properties of this quantization are recalled in the appendix of [16]. In particular, the quantization of these cutoffs preserves the eigenfunctions of the Laplacian:

Proposition 5.2. [4] For any fixed $L>0$, there exists $\hbar_{L}$ such that for any $\hbar \leq \hbar_{L}$, any $n \leq K_{\delta}|\log \hbar|$ and any sequence $\beta$ of length $n$, the Laplacian eigenstate verify

$$
\left\|\left(1-O p\left(\chi^{(n)}\right)\right) \pi_{\beta} \psi_{\hbar}\right\| \leq \hbar^{L}\left\|\psi_{\hbar}\right\| .
$$

\subsection{Applying Theorem A.1}

Let $\left\|\psi_{\hbar}\right\|=1$ be a fixed element of the sequence of eigenfunctions of the Laplacian defined earlier, associated to the eigenvalue $-\frac{1}{\hbar^{2}}$.

To get bound on the pressure of the suspension measure, the uncertainty principle should not be applied to the eigenvectors $\psi_{\hbar}$ directly but it will be 
applied several times. Precisely, we will apply it to each $P_{\gamma} \psi_{\hbar}:=P_{\gamma_{1}} P_{\gamma_{0}}(-\eta) \psi_{\hbar}$ where $\gamma=\left(\gamma_{0}, \gamma_{1}\right)$ varies in $\{1, \ldots, K\}^{2}$. In order to apply the uncertainty principle to $P_{\gamma} \psi_{\hbar}$, we introduce new families of quantum partitions corresponding to each $\gamma$.

Let $\gamma=\left(\gamma_{0}, \gamma_{1}\right)$ be an element of $\{1, \ldots, K\}^{2}$. We define $\gamma \cdot \alpha^{\prime}=\left(\gamma_{0}, \gamma_{1}, \alpha^{\prime}\right)$. Introduce the following families of indices:

$$
\begin{aligned}
I_{\hbar}(\gamma) & :=\left\{\left(\alpha^{\prime}\right): \gamma \cdot \alpha^{\prime} \in I^{\eta}(\hbar)\right\}, \\
K_{\hbar}(\gamma) & :=\left\{\left(\beta^{\prime}\right): \beta^{\prime} \cdot \gamma \in K^{\eta}(\hbar)\right\} .
\end{aligned}
$$

We underline that each sequence $\alpha$ of $I^{\eta}(\hbar)$ can be written under the form $\gamma \cdot \alpha^{\prime}$ where $\alpha^{\prime} \in I_{\hbar}(\gamma)$. The same works for $K^{\eta}(\hbar)$. Operators can be associated to these new families, for $\alpha^{\prime} \in I_{\hbar}(\gamma)$ and $\beta^{\prime} \in K_{\hbar}(\gamma)$,

$$
\begin{aligned}
& \tilde{\tau}_{\alpha^{\prime}}=P_{\alpha_{n}^{\prime}}(n \eta) \cdots P_{\alpha_{2}^{\prime}}(2 \eta), \\
& \tilde{\pi}_{\beta^{\prime}}=P_{\beta_{-n}^{\prime}}(-n \eta) \cdots P_{\beta_{-2}^{\prime}}(-2 \eta) .
\end{aligned}
$$

The families $\left(\tilde{\tau}_{\alpha^{\prime}}\right)_{\alpha^{\prime} \in I_{\hbar}(\gamma)}$ and $\left(\tilde{\pi}_{\beta^{\prime}}\right)_{\beta^{\prime} \in I_{\hbar}(\gamma)}$ form quantum partitions of identity [16].

Given these new quantum partitions of identity, the uncertainty principle should be applied for given initial conditions $\gamma=\left(\gamma_{0}, \gamma_{1}\right)$ at times 0 and 1 . We underline that, for $\alpha^{\prime} \in I_{\hbar}(\gamma)$ and $\beta^{\prime} \in K_{\hbar}(\gamma)$ :

$$
\tilde{\tau}_{\alpha^{\prime}} U^{-\eta} P_{\gamma}=\tau_{\gamma \cdot \alpha^{\prime}} U^{-\eta} \text { and } \tilde{\pi}_{\beta^{\prime}} P_{\gamma}=\pi_{\beta^{\prime} \cdot \gamma},
$$

where $\gamma \cdot \alpha^{\prime} \in I^{\eta}(\hbar)$ and $\beta^{\prime} \cdot \gamma \in K^{\eta}(\hbar)$ by definition. Equality (34) justifies that the definitions of $\tau$ and $\pi$ were slightly different (see (12) and (13)). It is due to the fact that we want to compose $\tilde{\tau}$ and $\tilde{\pi}$ with the same operator $P_{\gamma}$.

Suppose now that $\left\|P_{\gamma} \psi_{\hbar}\right\|$ is not equal to 0 . We apply the quantum uncertainty principle A.1 using that

- $\left(\tilde{\tau}_{\alpha^{\prime}}\right)_{\alpha^{\prime} \in I_{\hbar}(\gamma)}$ and $\left(\tilde{\pi}_{\beta^{\prime}}\right)_{\beta^{\prime} \in K_{\hbar}(\gamma)}$ are partitions of identity;

- the cardinal of $I_{\hbar}(\gamma)$ and $K_{\hbar}(\gamma)$ is bounded by $\mathcal{N} \simeq \hbar^{-K_{0}}$ where $K_{0}$ is some fixed positive number (depending on the cardinality of the partition $K$, on $a_{0}$, on $b_{0}$ and $\left.\eta\right)$;

- $\mathrm{Op}\left(\chi^{\left(k^{\prime}\right)}\right)$ is a family of bounded operators $O_{\beta^{\prime}}$ (where $k^{\prime}$ is the length of $\left.\beta^{\prime}\right)$;

- the constants $W_{\gamma \cdot \alpha^{\prime}}^{+}$and $W_{\beta . \gamma}^{-}$are bounded by $\hbar^{-\frac{b_{0}}{2 \epsilon_{0}}}$;

- the parameter $\delta^{\prime}$ can be taken equal to $\left\|P_{\gamma} \psi_{\hbar}\right\|^{-1} \hbar^{L}$ where $L$ is such that $\hbar^{L-K_{0}-\frac{b_{0}}{\epsilon_{0}}} \ll e^{2 k \eta \epsilon \epsilon_{0}} \hbar^{-1 / 2-c \delta_{0}}$ for every $k \ll \frac{1}{\epsilon \eta}|\log \hbar|$ (see Proposition 5.2 and the upper bound in Theorem 4.1);

- $U^{-\eta}$ is an isometry;

- $\tilde{\psi}_{\hbar}:=\frac{P_{\gamma} \psi_{\hbar}}{\left\|P_{\gamma} \psi_{\hbar}\right\|}$ is a normalized vector.

Applying the uncertainty principle A.1 for quantum pressures, one gets:

Corollary 5.3. Suppose that $\left\|P_{\gamma} \psi_{\hbar}\right\|$ is not equal to 0 . Then, one has

$$
p_{\tilde{\tau}}\left(U^{-\eta} \tilde{\psi}_{\hbar}\right)+p_{\tilde{\pi}}\left(\tilde{\psi}_{\hbar}\right) \geq-2 \log \left(c_{\chi}^{\gamma}\left(U^{-\eta}\right)+\hbar^{L-K_{0}-\frac{b_{0}}{\epsilon_{0}}}\left\|P_{\gamma} \psi_{\hbar}\right\|^{-1}\right),
$$

where $c_{\chi}^{\gamma}\left(U^{-\eta}\right)=\max _{\alpha^{\prime} \in I_{\hbar}(\gamma), \beta^{\prime} \in K_{\hbar}(\gamma)}\left(W_{\gamma \cdot \alpha^{\prime}}^{+} W_{\beta^{\prime} \cdot \gamma}^{-}\left\|\tilde{\tau}_{\alpha^{\prime}} U^{-\eta} \tilde{\pi}_{\beta^{\prime}}^{*} O p\left(\chi^{\left(k^{\prime}\right)}\right)\right\|\right)$. 
Under this form, the quantity $\left\|P_{\gamma} \psi_{\hbar}\right\|^{-1}$ appears several times and we would like to get rid of it. First, remark that the quantity $c_{\chi}^{\gamma}\left(U^{-\eta}\right)$ can be bounded by

$$
c_{\chi}\left(U^{-\eta}\right):=\max _{(*)}\left(W_{\gamma \cdot \alpha^{\prime}}^{+} W_{\beta^{\prime} \cdot \gamma}^{-}\left\|\tilde{\tau}_{\alpha^{\prime}} U^{-\eta} \tilde{\pi}_{\beta^{\prime}}^{*} \mathrm{Op}\left(\chi^{\left(k^{\prime}\right)}\right)\right\|\right),
$$

where $(*)$ means $\left(\gamma \in\{1, \ldots, K\}^{2}\right.$ and $\left.\alpha^{\prime} \in I_{\hbar}(\gamma), \beta^{\prime} \in K_{\hbar}(\gamma)\right)$. This last quantity is independent of $\gamma$. Then, one has the following lower bound:

$$
\begin{aligned}
& -2 \log \left(c_{\chi}^{\gamma}\left(U^{-\eta}\right)+\hbar^{L-K_{0}-\frac{b_{0}}{\epsilon_{0}}}\left\|P_{\gamma} \psi_{\hbar}\right\|^{-1}\right) \\
& \geq-2 \log \left(c_{\chi}\left(U^{-\eta}\right)+\hbar^{L-K_{0}-\frac{b_{0}}{\epsilon_{0}}}\right)+2 \log \left\|P_{\gamma} \psi_{\hbar}\right\|^{2} .
\end{aligned}
$$

as $\left\|P_{\gamma} \psi_{\hbar}\right\| \leq 1$. Now that we have given an alternative lower bound, we rewrite the entropy term $h_{\tilde{\tau}}\left(U^{-\eta} \tilde{\psi}_{\hbar}\right)$ of the quantum pressure $p_{\tilde{\tau}}\left(U^{-\eta} \tilde{\psi}_{\hbar}\right)$ as follows:

$$
\begin{aligned}
h_{\tilde{\tau}}\left(U^{-\eta} \tilde{\psi}_{\hbar}\right)= & -\sum_{\alpha^{\prime} \in I_{\hbar}(\gamma)}\left\|\tilde{\tau}_{\alpha^{\prime}} U^{-\eta} \tilde{\psi}_{\hbar}\right\|^{2} \log \left\|\tilde{\tau}_{\alpha^{\prime}} U^{-\eta} P_{\gamma} \psi_{\hbar}\right\|^{2} \\
& +\sum_{\alpha^{\prime} \in I_{\hbar}(\gamma)}\left\|\tilde{\tau}_{\alpha^{\prime}} U^{-\eta} \tilde{\psi}_{\hbar}\right\|^{2} \log \left\|P_{\gamma} \psi_{\hbar}\right\|^{2} .
\end{aligned}
$$

Using the fact that $\psi_{\hbar}$ is an eigenvector of $U^{\eta}$ and that $\left(\tilde{\tau}_{\alpha^{\prime}}\right)_{\alpha^{\prime} \in I_{\hbar}(\gamma)}$ is a partition of identity, one has:

$$
h_{\tilde{\tau}}\left(U^{-\eta} \tilde{\psi}_{\hbar}\right)=-\frac{1}{\left\|P_{\gamma} \psi_{\hbar}\right\|^{2}} \sum_{\alpha^{\prime} \in I_{\hbar}(\gamma)}\left\|\tau_{\gamma \cdot \alpha^{\prime}} \psi_{\hbar}\right\|^{2} \log \left\|\tau_{\gamma \cdot \alpha^{\prime}} \psi_{\hbar}\right\|^{2}+\log \left\|P_{\gamma} \psi_{\hbar}\right\|^{2}
$$

The same holds for the entropy term $h_{\tilde{\pi}}\left(\tilde{\psi}_{\hbar}\right)$ of the quantum pressure $p_{\tilde{\pi}}\left(\tilde{\psi}_{\hbar}\right)$ (using here equality (34)):

$$
h_{\tilde{\pi}}\left(\tilde{\psi}_{\hbar}\right)=-\frac{1}{\left\|P_{\gamma} \psi_{\hbar}\right\|^{2}} \sum_{\beta^{\prime} \in K_{\hbar}(\gamma)}\left\|\pi_{\beta^{\prime} \cdot \gamma} \psi_{\hbar}\right\|^{2} \log \left\|\pi_{\beta^{\prime} \cdot \gamma} \psi_{\hbar}\right\|^{2}+\log \left\|P_{\gamma} \psi_{\hbar}\right\|^{2} .
$$

Combining these last two equalities with (36), we find that

$$
\begin{aligned}
& -\sum_{\alpha^{\prime} \in I_{\hbar}(\gamma)}\left\|\tau_{\gamma \cdot \alpha^{\prime}} \psi_{\hbar}\right\|^{2} \log \left\|\tau_{\gamma \cdot \alpha^{\prime}} \psi_{\hbar}\right\|^{2}-2 \sum_{\alpha^{\prime} \in I_{\hbar}(\gamma)}\left\|\tau_{\gamma \cdot \alpha^{\prime}} \psi_{\hbar}\right\|^{2} \log W_{\gamma \cdot \alpha^{\prime}}^{+} \\
& \quad-\sum_{\beta^{\prime} \in K_{\hbar}(\gamma)}\left\|\pi_{\beta^{\prime} \cdot \gamma} \psi_{\hbar}\right\|^{2} \log \left\|\pi_{\beta^{\prime} \cdot \gamma} \psi_{\hbar}\right\|^{2}-2 \sum_{\beta^{\prime} \in K_{\hbar}(\gamma)}\left\|\pi_{\beta^{\prime} \cdot \gamma} \psi_{\hbar}\right\|^{2} \log W_{\beta^{\prime} \cdot \gamma}^{-} \\
& \quad \geq-2\left\|P_{\gamma} \psi_{\hbar}\right\|^{2} \log \left(c_{\chi}\left(U^{-\eta}\right)+\hbar^{L-K_{0}-\frac{b_{0}}{\epsilon_{0}}}\right)
\end{aligned}
$$

This expression is very similar to the definition of the quantum pressure. We also underline that this lower bound is trivial in the case where $\left\|P_{\gamma} \psi_{\hbar}\right\|$ is equal to 0 . Using the following numbers:

$$
c_{\gamma \cdot \alpha^{\prime}}=c_{\beta^{\prime} \cdot \gamma}=c_{\gamma}=\frac{f(\gamma)}{\sum_{\gamma^{\prime} \in\{1, \ldots, K\}^{2}} f\left(\gamma^{\prime}\right)\left\|P_{\gamma^{\prime}} \psi_{\hbar}\right\|^{2}},
$$

one can derive, as in [16], the following property: 
Corollary 5.4. One has:

$$
p\left(\bar{\mu}_{\hbar}^{\bar{\Sigma}_{+}}, \overline{\mathcal{C}}_{\hbar}^{+}\right)+p\left(\bar{\mu}_{\hbar}^{\bar{\Sigma}_{-}}, \overline{\mathcal{C}}_{\hbar}^{-}\right) \geq-2 \log \left(c_{\chi}\left(U^{-\eta}\right)+\hbar^{L-K_{0}-\frac{b_{0}}{\epsilon_{0}}}\right)-C,
$$

where $C:=\log \left(\max _{\gamma} c_{\gamma}\right)$.

As expected, by a careful use of the entropic uncertainty principle, we have been able to obtain a lower bound on the pressures of the measures $\bar{\mu}_{\hbar}^{\bar{\Sigma}_{+}}$ and $\bar{\mu}_{\hbar}^{\bar{\Sigma}_{-}}$.

\subsection{The Conclusion}

To conclude the proof of Proposition 5.1, we use Theorem 4.1 to give an upper bound on $c_{\chi}\left(U^{-\eta}\right)$. From our assumption on $L$, we know that $\hbar^{L-K_{0}-\frac{b_{0}}{\epsilon_{0}}} \ll$ $c_{\chi}\left(U^{-\eta}\right)$. As $k \eta \leq n_{E}(\hbar) / \epsilon_{0}$, we also have that

$$
c_{\chi}\left(U^{-\eta}\right) \leq C_{\mathcal{K}}\left(\epsilon, \eta, \epsilon_{0}\right) \hbar^{-\frac{1}{2}-c \delta_{0}} e^{4 \epsilon n_{E}(\hbar)} .
$$

For $\delta_{0}$ small enough, we find the expected property.

\section{Acknowledgements}

I would like to sincerely thank my advisor Nalini Anantharaman for introducing me to this question and for encouraging me to extend the result from [16] to nonpositively curved surfaces. I also thank her for many helpful discussions about this subject.

\section{Appendix A. Uncertainty Principle for the Quantum Pressure}

In [4], generalizations of the entropic uncertainty principle were derived for quantum pressures. We saw that the use of this thermodynamic formalism was crucial in our proof and we recall in this section the main results from [4, Sect. 6] on quantum pressures. Consider two partitions of identity $\left(\pi_{k}\right)_{k=1}^{\mathcal{N}}$ and $\left(\tau_{j}\right)_{j=1}^{\mathcal{M}}$ on $L^{2}(M)$, i.e.

$$
\sum_{k=1}^{\mathcal{N}} \pi_{k}^{*} \pi_{k}=\operatorname{Id}_{L^{2}(M)} \quad \text { and } \quad \sum_{j=1}^{\mathcal{M}} \tau_{j}^{*} \tau_{j}=\operatorname{Id}_{L^{2}(M)}
$$

We also introduce two families of positive numbers: $\left(V_{k}\right)_{k=1}^{\mathcal{N}}$ and $\left(W_{j}\right)_{j=1}^{\mathcal{M}}$. We denote $A:=\max _{k} V_{k}$ and $B:=\max _{j} W_{j}$. One can then introduce the quantum pressures associated to these families, for a normalized vector $\psi$ in $L^{2}(M)$,

$$
p_{\pi}(\psi):=-\sum_{k=0}^{\mathcal{N}}\left\|\pi_{k} \psi\right\|_{L^{2}(M)}^{2} \log \left\|\pi_{k} \psi\right\|_{L^{2}(M)}^{2}-2 \sum_{k=0}^{\mathcal{N}}\left\|\pi_{k} \psi\right\|_{L^{2}(M)}^{2} \log V_{k}
$$

and

$$
p_{\tau}(\psi):=-\sum_{j=0}^{\mathcal{M}}\left\|\tau_{j} \psi\right\|_{L^{2}(M)}^{2} \log \left\|\tau_{j} \psi\right\|_{L^{2}(M)}^{2}-2 \sum_{j=0}^{\mathcal{M}}\left\|\tau_{j} \psi\right\|_{L^{2}(M)}^{2} \log W_{j} .
$$

The main result on these quantities that was derived in [4] was Theorem 6.5: 
Theorem A.1. Under the previous setting, suppose $\mathcal{U}$ is an isometry of $L^{2}(M)$ and suppose $\left(O_{k}\right)_{k=1}^{\mathcal{N}}$ is a family of bounded operators. Let $\delta^{\prime}$ be a positive number and $\psi$ be a vector in $\mathcal{H}$ of norm 1 such that

$$
\left\|\left(I d-O_{k}\right) \pi_{k} \psi\right\|_{L^{2}(M)} \leq \delta^{\prime} .
$$

Then, one has

$$
p_{\tau}(\mathcal{U} \psi)+p_{\pi}(\psi) \geq-2 \log \left(c_{O}(\mathcal{U})+\max (\mathcal{N}, \mathcal{M}) A B \delta^{\prime}\right),
$$

where $c_{O}(\mathcal{U}):=\sup _{j, k}\left\{V_{k} W_{j}\left\|\tau_{j} \mathcal{U} \pi_{k}^{*} O_{k}\right\|\right\}$.

\section{Appendix B. Kolmogorov-Sinai Entropy}

Let us recall a few facts about Kolmogorov-Sinai (or metric) entropy that can be found for example in [22]. Let $(X, \mathcal{B}, \mu)$ be a measurable probability space, $I$ a finite set and $P:=\left(P_{\alpha}\right)_{\alpha \in I}$ a finite measurable partition of $X$, i.e. a finite collection of measurable subsets that forms a partition. Each $P_{\alpha}$ is called an atom of the partition. Assuming $0 \log 0=0$, one defines the entropy of the partition as:

$$
H(\mu, P):=-\sum_{\alpha \in I} \mu\left(P_{\alpha}\right) \log \mu\left(P_{\alpha}\right) \geq 0 .
$$

Given two measurable partitions $P:=\left(P_{\alpha}\right)_{\alpha \in I}$ and $Q:=\left(Q_{\beta}\right)_{\beta \in K}$, one says that $P$ is a refinement of $Q$ if every element of $Q$ can be written as the union of elements of $P$ and it can be shown that $H(\mu, Q) \leq H(\mu, P)$. Otherwise, one denotes $P \vee Q:=\left(P_{\alpha} \cap Q_{\beta}\right)_{\alpha \in I, \beta \in K}$ their join (which is still a partition) and one has $H(\mu, P \vee Q) \leq H(\mu, P)+H(\mu, Q)$ (subadditivity property). Let $T$ be a measure preserving transformation of $X$. The $n$-refined partition $\vee_{i=0}^{n-1} T^{-i} P$ of $P$ with respect to $T$ is then the partition made of the atoms $\left(P_{\alpha_{0}} \cap \cdots \cap T^{-(n-1)} P_{\alpha_{n-1}}\right)_{\alpha \in I^{n}}$. We define the entropy with respect to this refined partition:

$$
H_{n}(\mu, T, P):=\sum_{|\alpha|=n} S\left(\mu\left(P_{\alpha_{0}} \cap \cdots \cap T^{-(n-1)} P_{\alpha_{n-1}}\right)\right),
$$

where $S(x):=-x \log x$. Using the subadditivity property of entropy, we have for any integers $n$ and $m$ :

$$
\begin{aligned}
H_{n+m}(\mu, T, P) & \leq H_{n}(\mu, T, P)+H_{m}\left(\mu \circ T^{-n}, T, P\right) \\
& =H_{n}(\mu, T, P)+H_{m}(\mu, T, P) .
\end{aligned}
$$

For the last equality, it is important to underline that we really use the $T$-invariance of the measure $\mu$. A classical argument for subadditive sequences allows us to define the following quantity:

$$
h_{K S}(\mu, T, P):=\lim _{n \rightarrow \infty} \frac{H_{n}(\mu, T, P)}{n} .
$$

It is called the Kolmogorov Sinai entropy of $(T, \mu)$ with respect to the partition $P$. The Kolmogorov Sinai entropy $h_{K S}(\mu, T)$ of $(\mu, T)$ is then defined as the supremum of $h_{K S}(\mu, T, P)$ over all partitions $P$ of $X$. 
A property of entropy we used in the paper is the so-called Abramov property [1]. Using the notations of the article, one has

$$
h_{K S}\left(\mu^{\Sigma_{+}}, \sigma_{+}\right)=\left(\int_{\Sigma_{+}} f_{+} \mathrm{d} \mu^{\Sigma^{+}}\right) h_{K S}\left(\bar{\mu}^{\bar{\Sigma}_{+}}, \bar{\sigma}_{+}\right) .
$$

\section{References}

[1] Abramov, L.M.: On the entropy of a flow. Transl. AMS 49, 167-170 (1966)

[2] Anantharaman, N.: Entropy and the localization of eigenfunctions. Ann. Math. 168, 435-475 (2008)

[3] Anantharaman, N., Koch, H., Nonnenmacher, S.: Entropy of eigenfunctions. International Congress of Mathematical Physics, arXiv:0704.1564

[4] Anantharaman, N., Nonnenmacher, S.: Half-delocalization of eigenfunctions for the Laplacian on an Anosov manifold. Ann. Inst. Fourier 57, 2465-2523 (2007)

[5] Ballmann, W., Brin, M., Burns, K.: On surfaces with no conjugate points. J. Differ. Geom. 25, 249-273 (1987)

[6] Barreira, L., Pesin, Y.: Lectures on Lyapunov exponents and smooth ergodic theory. Proc. Symp. Pure Math. 69, 3-89 (2001)

[7] Colin de Verdière, Y.: Ergodicité et fonctions propres du Laplacien. Comm. Math. Phys. 102, 497-502 (1985)

[8] Dimassi, M., Sjöstrand, J.: Spectral Asymptotics in the Semiclassical Limit. Cambridge University Press, Cambridge (1999)

[9] Donnelly, H.: Quantum unique ergodicity. Proc. Am. Math. Soc. 131, 2945-2951 (2002)

[10] Eberlein, P.: When is a geodesic flow of Anosov type I. J. Differ. Geom. 8, 437-463 (1973)

[11] Eberlein, P.: Geodesic flows in manifolds of nonpositive curvature. Proc. Symp. Pure Math. 69, 525-571 (2001)

[12] Freire, A., Mañé, R.: On the entropy of the geodesic flow for manifolds without conjugate points. Inv. Math. 69, 375-392 (1982)

[13] Green, L.: Geodesic instability. Proc. Am. Math. Soc. 7, 438-448 (1956)

[14] Hassell, A.: Ergodic billiards that are not quantum unique ergodic. With an appendix by A. Hassell and L. Hillairet. Ann. Math. 171(1):605-618 (2010)

[15] Ledrappier, F., Young, L.-S.: The metric entropy of diffeomorphisms I. Characterization of measures satisfying Pesin's entropy formula. Ann. Math. 122, 509539 (1985)

[16] Rivière, G.: Entropy of semiclassical measures in dimension 2. Duke Math. J. (2010, to appear). arXiv:0809.0230

[17] Rudnick, Z., Sarnak, P.: The behaviour of eigenstates of arithmetic hyperbolic manifolds. Comm. Math. Phys. 161, 195-213 (1994)

[18] Ruelle, D.: An inequality for the entropy of differentiable maps. Bol. Soc. Bras. Mat. 9, 83-87 (1978)

[19] Ruggiero, R.O.: Dynamics and global geometry of manifolds without conjugate points. Ensaios Mate. 12, Soc. Bras. Mate. (2007) 
[20] Shnirelman, A.: Ergodic properties of eigenfunctions. Usp. Math. Nauk. 29, 181$182(1974)$

[21] Sjöstrand, J., Zworski, M.: Asymptotic distribution of resonances for convex obstacles. Acta Math. 183, 191-253 (1999)

[22] Walters, P.: An Introduction to Ergodic Theory. Springer, Berlin (1982)

[23] Zelditch, S.: Uniform distribution of the eigenfunctions on compact hyperbolic surfaces. Duke Math. J. 55, 919-941 (1987)

Gabriel Rivière

Centre de Mathématiques Laurent Schwartz (UMR 7640)

École Polytechnique

91128 Palaiseau Cedex, France

e-mail: gabriel.riviere@polytechnique.edu

Communicated by Viviane Baladi.

Received: December 4, 2009.

Accepted: July 19, 2010. 